\title{
Decaimento de geometria e medidas invariantes para polinômios cúbicos
}

\section{Liane Bordignon}

TESE APRESENTADA

$\mathrm{AO}$

INSTITUTO DE MATEMÁTICA E ESTATÍSTICA

DA

UNIVERSIDADE DE SÃO PAULO

PARA

OBTENÇÃO DO GRAU DE DOUTOR

EM

MATEMÁTICA

Área de Concentração: Sistemas Dinâmicos

Orientador: Prof. Dr. Edson Vargas

Durante a elaboração deste trabalho a autora recebeu apoio financeiro da CAPES. 


\title{
Decaimento de geometria e medidas invariantes para polinômios cúbicos
}

\author{
Este exemplar corresponde à redação \\ final da tese devidamente corrigida e \\ defendida por Liane Bordignon \\ e aprovada pela comissão julgadora.
}

São Paulo, 20 de Março de 2003.

Banca examinadora:

- Prof. Dr. Edson Vargas (Orientador) - IME - USP

- Prof. Dr. Eduardo Colli - IME - USP

- Prof. Dr. Marco Antônio Teixeira - IMECC - UNICAMP

- Prof. Dr. Carlos Gustavo Tamm de Araújo Moreira - IMPA

- Prof. Dr. Márcio Lima do Nascimento - UFPA 


\title{
Resumo
}

Neste trabalho são estudados polinômios cúbicos bimodais Fibonacci, os quais exibem decaimento de geometria. Demonstra-se que tais polinômios induzem expansão, não possuem atrator selvagem e possuem uma medida de probabilidade invariante absolutamente contínua em relação à medida de Lebesgue. Os polinômios de Fibonacci possuem pontos críticos com forte recorrência o que os torna especialmente interessantes entre as aplicações bimodais.

\begin{abstract}
This work deals with Fibonacci bimodal cubic polynomial maps which have decay of geometry. It is proved that these maps induce expansion, have no wild attractor and have an absolutely continuous invariant probability measure. The Fibonacci bimodal polynomial maps have strongly recurrent critical points which makes them especially interesting.
\end{abstract}




\section{Sumário}

$\begin{array}{ll}\text { Resumo } & 3\end{array}$

$\begin{array}{ll}\text { Abstract } & 3\end{array}$

$\begin{array}{ll}\text { Introdução } & 6\end{array}$

1 Principais Resultados 9

1.1 Conceitos e Fatos Básicos ... . . . . . . . . . . . . 9 9

1.2 Principais Resultados . . . . . . . . . . . . . . . . . 13

2 Ferramentas Básicas $\quad \mathbf{1 5}$

2.1 Princípios de Koebe e Outros Fatos . . . . . . . . . . . . . . . 15

2.2 Teorema do Folclore . . . . . . . . . . . . . . . . . 18

2.3 Média Temporal . . . . . . . . . . . . . . . . . 20

3 Polinômios de Fibonacci $\quad 22$

3.1 Existência de Polinômios Fibonacci . . . . . . . . . . . . . . 22

3.2 Fibonacci Cúbico, Propriedades Topológicas . . . . . . . . . . . 27

4 Hiperbolicidade Induzida 30

4.1 Fibonacci Induz Markov . . . . . . . . . . . . . . . . . . . . 30

4.2 Decaimento de Geometria e Hiperbolicidade Induzida . . . . . . . 36

5 Probabilidade Invariante 44 
5.1 Expansividade dos Ramos Críticos . . . . . . . . . . . . . . 44

5.2 Somabilidade nas Órbitas Críticas . . . . . . . . . . . . . 47

5.3 A Média Temporal . . . . . . . . . . . . . . . . . 50

Referências Bibliográficas $\quad 56$ 


\section{Introdução}

Um dos temas mais relevantes em sistemas dinâmicos unidimensionais diz respeito a aspectos métricos dos sistemas determinados por iterações de aplicações multimodais $f:[0,1] \rightarrow[0,1]$. No contexto mais geral e razoável assumimos que $f$ é de classe $C^{2}$ e possui um número finito de pontos críticos, todos de ordem finita. Neste tipo de sistema dinâmico uma região expansiva onde $f$ possui derivada. grande interage com uma região fortemente contrativa próxima aos seus pontos críticos. Decidir qual destes dois comportamentos, o expansivo ou o contrativo, será predominante à medida que $f$ é iterada torna-se uma questão fundamental. A predominância de um destes comportamentos pode ser medida através, por exemplo, da análise dos expoentes de Liapunov dos valores críticos, da existência de uma aplicação de Markov expansora induzida por $f$ (expansão ou hiperbolicidade induzida), da medida de Lebesgue da bacia de atração dos conjuntos $\omega$-limite dos pontos críticos ou da existência de medida de probabilidade invariante e absolutamente contínua (abreviadamente, p.i.a.c.).

No caso de polinômios quadráticos reais, a saber $x \mapsto \lambda x(1-x)$, onde $\lambda$ é um parâmetro real que varia em $[2,4]$, sabemos que existem dois tipos de comportamentos mais importantes: existe uma p.i.a.c. ou o polinômio é regular (isto é: todos os pontos periódicos são hiperbólicos e o ponto crítico $c=1 / 2$ é atraído por um ponto periódico atrator. Estes polinômios também são chamados de $h i$ perbólicos). Os parâmetros $\lambda$ correspondentes a estes dois comportamentos formam um conjunto de medida de Lebesgue total em [2,4], veja [MN]. Os parâmetros correspondentes à hiperbolicidade são facilmente detectáveis através da periodicidade ou não da seqüência de kneading enquanto que a existência de uma p.i.a.c. depende de uma análise mais profunda. Inicialmente imaginou-se que seria suficiente que o polinômio quadrático fosse não-renormalizável (em geral uma aplicação multimodal é dita não-renormalizável se não possuir intervalos periódicos cuja órbita positiva contenha pelo menos um ponto crítico). Na ausência de pontos periódicos atratores isto garante a existência de uma conjugação topológica com uma aplicação expansiva do tipo tenda $x \mapsto \min \{s x, s(x-1)\}$. Comprovou-se então que este não é o caso, veja [J] e [HK1]. 
A frequência com que os pontos críticos de uma aplicação multimodal $f$ retornam próximos deles mesmos, ou seja, a capacidade de recorrência destes pontos é um dos fatores mais importantes para detectarmos a existência de uma p.i.a.c. Então, aplicações de primeiro retorno se tornam uma ferramenta muito útil nesta análise. Consideramos a situação em que $f$ possui pontos críticos recorrentes e escolhemos intervalos convenientes contendo estes pontos. Usualmente escolhemos intervalos determinados pelas órbitas negativas dos pontos fixos de $f$. No caso em que $f$ é um polinômio quadrático como acima existe um único ponto fixo $p \in(0,1)$ e então consideramos $\phi_{0}$, a aplicação de primeiro retorno ao intervalo $I_{0}:=\left[p^{*}, p\right]$, onde $p^{*}$ é o único ponto tal que $f\left(p^{*}\right)=f(p)$. Se o ponto crítico $c$ é recorrente a componente conexa do domínio de $\phi_{0}$ que contem $c$ é não-vazia e então denotamo-la por $I_{1}$. Passamos então a considerar $\phi_{1}$, a aplicação de primeiro retorno a $I_{1}$. Sendo $c$ recorrente podemos definir indutivamente a seqüência de intervalos $I_{n}$ juntamente com as suas respectivas aplicações de primeiro retorno $\phi_{n}$. O tamanho relativo $\left|I_{n+1}\right| /\left|I_{n}\right|$ está relacionado com a expansividade de $\phi_{n \mid I_{n+1}}$ e o número $k_{n}$ de iteradas tais que $\phi_{n}^{k_{n}}(c)=\phi_{n+1}(c)$ é uma medida da. capacidade de recorrência de $c$. Se $k_{n}=1$ resulta que $\phi_{n}(c) \in I_{n+1}$ e então dizemos que $\phi_{n}(c)$ é um retorno central. Se $k_{n}=\ldots=k_{n+j}=1$ e $k_{n+j+1} \neq 1$ resulta que $\phi_{n}(c)=\ldots=\phi_{n+j+1}(c) \in I_{n+j+1} \backslash I_{n+j+2}$ e neste caso dizemos que $\phi_{n}, \ldots, \phi_{n+j+1}$ é uma cascata de retornos centrais. Estas cascatas estão associadas a uma forte recorrência. O exemplo de Jonhson $[\mathrm{J}]$ prova a existência de um polinômio quadrático não-renormalizável que não possui p.i.a.c. Ao mesmo tempo detecta-se a presença de cascatas de retornos centrais arbitrariamente longas indicando uma forte recorrência do ponto crítico. No caso em as cascatas de retornos centrais têm comprimento limitado ocorre que $\left|I_{n+1}\right| /\left|I_{n}\right|$ tende exponencialmente para zero quando $n \rightarrow \infty$ o que chamamos de decaimento exponencial de geometria. Provou-se em $[\mathrm{MN}]$ que isto garante a convergência da série

$$
\sum_{i=0}^{\infty}\left|D f^{i}(f(c))\right|^{-1 / 2}
$$

e esta convergência, como provado em [NSt], garante a existência de uma p.i.a.c. Analogamente, no caso em que $f$ é uma aplicação multimodal com pontos críticos $c_{1}, c_{2}, \ldots, c_{l}$, todos não-planos, cuja ordem mais alta é $l_{m}$, foi provado em [BSt] que se a soma

$$
\sum_{j=1}^{l} \sum_{i=0}^{\infty}\left|D f^{i}\left(f\left(c_{j}\right)\right)\right|^{-1 / l_{m}}
$$

converge, então $f$ possui p.i.a.c.. Neste trabalho consideraremos os polinômios cúbicos $f(x)=a x^{3}+b x^{2}+(1-a-b) x$ que são bimodais (possuem dois extremos locais em $(0,1))$ para os quais introduziremos os conceitos de retornos centrais e 
decaimento de geometria. Então conjecturamos que a ausência de retornos centrais implica no decaimento exponencial de geometria e este por sua vez garante expansão induzida, não existência de atratores selvagens (veja a definição na próxima seção) e existência de uma p.i.a.c. ergódica com entropia positiva. Não chegaremos a provar esta conjectura em geral mas o faremos para uma situação que julgamos ser a mais delicada.

No primeiro capítulo descreveremos os principais resultados do trabalho e os conceitos básicos. No segundo capítulo, são citadas as ferramentas que usaremos no decorrer do trabalho. A seguir, no terceiro capítulo, descreveremos e daremos características topológicas dos polinômios cúbicos bimodais de Fibonacci. No quarto capítulo construiremos uma partição de [0,1] e uma aplicação de Markov hiperbólica induzida por um polinômio cúbico de Fibonacci $f$, definida nos intervalos desta partição. Com isto, demonstraremos que $f$ induz expansão e não tem atrator selvagem. No quinto capítulo, usaremos a aplicação de Markov construída e o decaimento de geometria para garantir condições de somabilidade que implicam a existência de uma p.i.a.c. para $f$. 


\section{Capítulo 1}

\section{Principais Resultados}

\subsection{Conceitos e Fatos Básicos}

Seja $f:[0,1] \rightarrow[0,1]$ uma aplicação contínua. Esta aplicação é chamada multimodal se o intervalo $[0,1]$ possui uma partição com um número finito de intervalos não-degenerados restrita aos quais $f$ é estritamente monótona. Se $f$ é derivável os extremos destes intervalos que estão em $(0,1)$ serão pontos críticos extremos locais de $f$. No caso em que esta partição possui apenas dois intervalos $[0, c]$ e $[c, 1]$ a aplicação $f$ é dita unimodal. Este é o caso dos polinômios quadráticos. Se esta partição possui três intervalos, $[0, c],[c, d]$ e $[d, 1]$, a aplicação $f$ é dita bimodal.

A presença de pontos extremos locais para uma aplicação mutimodal $f$ contribui para a complexidade da sua dinâmica. No intuito de obter algumas informações podemos tentar eliminar estes extremos (e também os pontos críticos de inflexão) definindo uma outra aplicação que em certos intervalos coincide com iteradas de $f$. Isto nos leva ao conceito de aplicação de Markov topológica induzida por $f$ que introduzimos abaixo.

Definição 1.1. Seja $\mathcal{P}=\left\{N_{j}\right\}_{j=1}^{\kappa}(\kappa \leq \infty)$ uma coleção de intervalos $N_{j} \subset$ $\mathbb{R}$ não-degenerados e cujos interiores são dois a dois disjuntos. Uma aplicação $F: \cup_{j=1}^{\kappa} N_{j} \rightarrow \mathbb{R}$ é chamada de aplicação de Markov se cada ramo $F_{\mid N_{j}}$ é um homeomorfismo sobre a sua imagem a qual contém todos os intervalos $N_{i}$ tais que o interior de $N_{i} \cap F\left(N_{j}\right)$ é não-vazio. Dizemos que $F$ é de classe $C^{r}(r \geq 0)$ se cada ramo $F_{\mid N,}$ possui uma extensão para um difeomorfismo de classe $C^{r} \bar{d}$ efinido em um intervalo aberto que contém o fecho de $N_{j}$. A coleção de intervalos $F(\mathcal{P})=$ $\left\{F\left(N_{j}\right)\right\}_{j=1}^{\kappa}$ é chamada de partição imagem. Se cada ramo $F_{N_{j}}$ coincide com um iterado $f_{\mid N_{j}}^{n_{j}}$, para algum $n_{j}$, dizemos que $F$ é uma aplicação de Markov induzida por $f$. 
Uma aplicação de Markov induzida com propriedades apenas topológicas não é suficiente para garantir boas propriedades métricas para uma aplicação multimodal. Portanto introduzimos mais um conceito.

Definição 1.2. Uma aplicação de Markov $F: \cup_{j=1}^{\kappa} N_{j} \rightarrow \mathbb{R}$ de classe $C^{r}(r \geq 1)$ é dita hiperbólica em $[0,1]$ se:

1. O domínio de $F$ é uma união de intervalos abertos $\cup_{j=1}^{\kappa} N_{j}$ com medida de Lebesgue total em $[0,1]$.

2. A partição imagem $F(\mathcal{P})=\left\{F\left(N_{j}\right)\right\}_{j=1}^{\kappa}$ é uma coleção finita de intervalos limitados.

3. Existe $a>1$ tal que $|D F(x)| \geq a$, para todo $x \in \cup_{j=1}^{\kappa} N_{j}$.

4. Existe $K<\infty$ tal que $\frac{D F(x)}{D F(y)} \leq K$, para todo $x, y \in N_{j}$.

Dizemos que uma aplicação multimodal $f:[0,1] \rightarrow[0,1]$ de classe $C^{1}$ induz expansão ou induz hiperbolicidade se a mesma induz uma aplicação de Markov hiperbólica em $[0,1]$.

Um aspecto importante para a compreensão de um sistema dinâmico é a descrição dos seus atratores os quais podem ser topológicos ou métricos. Dado um conjunto compacto $\mathcal{A}$ positivamente invariante por $f$ (isto é $f(\mathcal{A}) \subset \mathcal{A}$ ) definimos a sua bacia de atração por

$$
B(\mathcal{A})=\{x \in[0,1] \text { tal que } \omega(x) \subset \mathcal{A}\} .
$$

O conjunto $\mathcal{A}$ é chamado de atrator topológico se

- $B(\mathcal{A})$ contém um conjunto de segunda categoria de Baire;

- dado um conjunto positivamente invariante $\mathcal{A}^{\prime}$ estritamente contido em $\mathcal{A}$, então $\overline{B(\mathcal{A})} \backslash \overline{B\left(\mathcal{A}^{\prime}\right)}$ é um conjunto de segunda categoria de Baire.

Sabemos que se $f$ é não-renormalizável e não possui atrator periódico então o seu atrator topológico é um intervalo invariante no qual $f$ é transitiva (veja [L] e [MSt]).

$\mathrm{O}$ conjunto $\mathcal{A}$ é chamado de atrator métrico se

- sua bacia de atração $B(\mathcal{A})$ tem medida de Lebesgue positiva; 
- se $\mathcal{A}^{\prime}$ é um conjunto positivamente invariante e estritamente contido em $\mathcal{A}$, então $B(\mathcal{A}) \backslash \mathcal{B}\left(\mathcal{A}^{\prime}\right)$ tem medida positiva.

Em [L] encontramos uma descrição detalhada de atratores métricos (que existem por $[\mathrm{M}]$ ) de aplicações multimodais de classe $C^{2}$. Para a classe de funções da qual trataremos neste trabalho podemos enunciar o seguinte teorema (veja [StV]):

Teorema 1.3. Seja $f:[0,1] \rightarrow[0,1]$ uma aplicação de classe $C^{3}$ com pontos críticos de ordem finita. Então

1. Todo conjunto minimal A tem medida de Lebesgue nula;

2. Há um número finito de compactos invariantes $A_{1}, A_{2}, \ldots, A_{k}$, cada um deles contendo um ponto critico, tais que $\cup B\left(A_{i}\right)$ tem medida total em $[0,1]$. Além disso, cada $A_{i}$ é uma órbita periódica atratora, um ciclo de intervalos tais que $\omega(x)=A_{i}$ para quase todo ponto $x \in A_{i}$ ou $A_{i}$ é um conjunto minimal contendo no mínimo um ponto crítico recorrente.

3. Se $A_{i}$ não é uma órbita periódica atratora então $f_{\mid B\left(A_{i}\right)}$ é ergódica em relação à medida de Lebesgue.

Se $\mathcal{A}$ é um atrator métrico que não é um atrator topológico então será chamado de atrator selvagem. A questão da existência ou não de atratores selvagens foi colocada por Milnor [M] e tem atraído a atenção de muitos pesquisadores. No caso de polinômios quadráticos, o que possui a combinatória de Fibonacci é o principal candidato a exibir um atrator selvagem. Como foi provado em [LM] isto de fato não ocorre. No entanto um polinômio unimodal real com ponto crítico suficientemente degenerado (a saber $x \mapsto x^{2 l}+c$ com $l$ suficientemente grande) exibindo a combinatória de Fibonacci exibe um atrator selvagem, veja [BKNSt].

Uma medida de probabilidade $\mu$ invariante por $f$ é dita ergódica se $\mu(X)=0$ ou $\mu(X)=1$ para todo Boreleano $X$ tal que $f(X) \subset X$. Por outro lado a aplicação $f$ é dita ergódica em relação à medida de Lebesgue (abreviadamente diremos apenas ergódica) se $|X|=0$ ou $|X|=1$ para todo Boreleano $X$ tal que $f^{-1}(X)=X$, onde $|A|$ é a medida de Lebesgue de um Boreleano $A$.

Neste trabalho estaremos interessados nos polinômios cúbicos reais descritos por $f(x)=f_{a b}(x)=a x^{3}+b x^{2}+(1-a-b) x$ que são bimodais. Observe que $f(0)=0$ e $f(1)=1$. Neste caso $c$ e $d$ são pontos críticos distintos que assumiremos serem recorrentes. E como a derivada de Schwarz

$$
S f(x)=\frac{f^{\prime \prime \prime}(x)}{f^{\prime}(x)}-\frac{3}{2}\left(\frac{f^{\prime \prime}(x)}{f^{\prime}(x)}\right)^{2}
$$


definida para todo $x \neq c, d$ é negativa podemos assumir também que $f$ não possui pontos periódicos atratores, veja [Si].

Os nossos principais resultados relacionam a existência de expansão induzida, a não existência de atratores selvagens e a existência de uma p.i.a.c. com propriedades da seqüência de aplicações de primeiro retorno que introduziremos a seguir. Consideramos aplicações de primeiro retorno associadas a certos pares de intervalos abertos cuja união contém $c$ e $d$. Escolhemos intervalos que são componentes conexas do complementar de um trecho finito da órbita negativa dos pontos fixos de $f$. Temos que $f$ é estritamente crescente em $[0, c]$, estritamente decrescente em $[c, d]$ e estritamente crescente em $[d, 1]$. Uma vez que estamos assumindo que os pontos críticos $c$ e $d$ são recorrentes resulta que $f$ possui um único ponto fixo $p$ em $(0,1)$ e de fato $p \in(c, d)$. Definimos então os intervalos $I_{0}:=\left(p_{c}, p\right)$ e $J_{0}:=\left(p, p_{d}\right)$, onde $p_{c} \in(0, c)$ é o único ponto tal que $f\left(p_{c}\right)=p$ e $p_{d} \in(d, 1)$ é o único ponto tal que $f\left(p_{d}\right)=p$. Definimos $\phi_{0}$, a aplicação de primeiro retorno a $I_{0} \cup J_{0}$ como composição de $f_{\mid I_{0} \cup J_{0}}$ com $\psi_{0}$, a aplicação de primeira entrada em $I_{0} \cup J_{0}$, veja [GS]. Para definirmos $\psi_{0}$ consideramos $g$ a qual é igual a $f$ em $\left[0, p_{c}\right] \cup\left[p_{d}, 1\right]$ e igual a identidade em $I_{0} \cup J_{0}$. Então definimos

$$
\psi_{0}(x):=\lim _{i \rightarrow \infty} g^{i}(x),
$$

para todo $x$ tal que este limite esteja bem definido. Note que o domínio de $\psi_{0}$ é uma união enumerável de intervalos abertos e dois a dois disjuntos. A restrição de $\psi_{0}$ a cada um destes intervalos, que chamamos ramo de $\psi_{0}$, é monótona e tem imagem igual a $I_{0}$ ou $J_{0}$. Então definimos $\phi_{0}:=\psi \circ f_{\mid I_{0} \cup J_{0}}$ cujo domínio $D\left(\phi_{0}\right)$ também é uma união enumerável de intervalos abertos e dois a dois disjuntos. Chamamos ramo de $\phi_{0}$ à restrição de $\phi_{0}$ a cada um destes intervalos. Dois destes ramos possuem pontos críticos e são chamados ramos críticos, são os ramos cujos domínios são os intervalos $I_{1}$ e $J_{1}$ que contém $c$ e $d$ respectivamente. Cada um destes ramos críticos é uma aplicação unimodal cuja imagem está contida em $I_{0}$ ou $J_{0}$. Cada um dos ramos de $\phi_{0}$ que não é crítico aplica o seu domínio monotonamente sobre $I_{0}$ ou $J_{0}$. De maneira análoga definimos $\phi_{1}$, a aplicação de primeiro retorno de $I_{1} \cup J_{1}$. Prosseguindo por indução obtemos duas seqüências de intervalos $I_{n}$ e $J_{n}$ e a aplicação de primeiro retorno de $I_{n} \cup J_{n}$ que denotamos por $\phi_{n}$. Os ramos de $\phi_{n}$ definidos em $I_{n+1}$ e $J_{n+1}$ são ramos críticos e unimodais cujas imagens estão contidas em $I_{n}$ ou $J_{n}$. Cada um dos ramos de $\phi_{n}$ que não é crítico aplica o seu domínio monotonamente sobre $I_{n}$ ou $J_{n}$.

Notamos que se $f$ é não-renormalizável (ou seja, $f$ não possui intervalo periódico cuja órbita positiva contenha $c$ ou $d$ ) então $\cap_{i=0}^{\infty} I_{i}=\{c\}$ e $\cap_{i=0}^{\infty} J_{i}=\{d\}$. Consideramos então as duas seqüências 


$$
\frac{\left|I_{n+1}\right|}{\left|I_{n}\right|} \text { e } \frac{\left|J_{n+1}\right|}{\left|J_{n}\right|}
$$

e dizemos que ocorre decaimento exponencial de geometria se estas seqüências tendem exponencialmente para zero quando $n \rightarrow \infty$.

Se $\phi_{n}(c) \in I_{n+1} \cup J_{n+1}$ ou $\phi_{n}(d) \in I_{n+1} \cup J_{n+1}$ dizemos que $\phi_{n}$ é um retorno central. Uma cascata de retornos centrais é uma seqüência $\phi_{n}, \ldots, \phi_{n+k}$ de retornos centrais consecutivos. Se $\phi_{n}, \ldots, \phi_{n+k}$ é uma cascata de retornos centrais consecutivos e $\phi_{n-1}, \phi_{n+k+1}$ não são retornos centrais dizemos que $k$ é o comprimento da cascata. No caso de aplicações unimodais como os polinômios quadráticos, assumindo que sejam não-renormalizáveis, as cascatas de retornos centrais podem ter comprimentos arbitrariamente longos mas são sempre finitos. Para aplicações bimodais como os polinômios cúbicos que estamos considerando, mesmo assumindo que sejam não-renormalizáveis, tais cascatas podem ter comprimento infinito. Conjecturamos que a ausência de retornos centrais é suficiente para garantir o decaimento exponencial de geometria que por sua vez é suficiente para garantir a existência de uma p.i.a.c. Neste trabalho provaremos esta última propriedade para um polinômio cúbico $f$ específico. Consideramos uma das situações mais delicadas devido ao fato de que os pontos críticos $c$ e $d$ são fortemente recorrentes.

Definição 1.4. Uma aplicação bimodal $f:[0,1] \rightarrow[0,1]$ é dita Fibonacci se para $n \geq 1$ verifica-se que $\phi_{n}(c)=f^{s_{n}}(c)$ e $\phi_{n}(d)=f^{s_{n}}(d)$, onde $c$ e d são os pontos críticos de $f, s_{0}=1, s_{1}=2$ e $s_{n+1}=s_{n}+s_{n-1}$ (ou seja, $s_{n}$ é a sequência de Fibonacci).

Se $f$ 'é Fibonacci não ocorrem retornos centrais mas verifica-se que $\phi_{n}^{2}(c)$ e $\phi_{n}^{2}(d)$ pertencem a $I_{n+1} \cup J_{n+1}$ o que poderia ser vista com uma das situações mais próximas de apresentar retornos centrais. As aplicações unimodais Fibonacci foram introduzidas em [HK2] (veja também [LM]) como o principal candidato a exibir atratores selvagens e desempenharam um papel fundamental em várias questões. Os polinômios Fibonacci bimodais foram introduzidos em [SV] onde se provou que os mesmos apresentam decaimento exponencial de geometria. No Capítulo 3, demostraremos, seguindo [SV] que existem polinômios cúbicos bimodais Fibonacci e que quaisquer dois destes são topologicamente conjugados.

\subsection{Principais Resultados}

Passamos a enunciar os nossos principais resultados.

Teorema A. Todo polinômio cúbico de Fibonacci induz expansão. 
Em particular o Teorema A garante a existência de uma aplicação de Markov $F$ induzida por $f$ e que possui uma p.i.a.c. ergódica que denotamos por $\mu$. Isto é suficiente para garantir que para $x$ q.t.p. (em relação à medida de Lebesgue) a órbita positiva $\operatorname{orb}^{+}(x)$ é densa em um intervalo. Com isto temos o seguinte teorema como conseqüência.

Teorema B. Os polinômio cúbicos de Fibonacci não possuem atrator selvagem.

A partir da p.i.a.c. associada à aplicação de Markov induzida $F$ sempre é possível definirmos uma medida (não necessariamente uma medida de probabilidade) invariante e absolutamente contínua para $f$. Na presença de decaimento exponencial de geometria podemos provar uma propriedade de somabilidade que nos leva ao teorema a seguir.

Teorema C. Todo polinômio cúbico de Fibonacci possui uma probabilidade invariante absolutamente contínua, ergódica e de entropia positiva.

Se $\nu$ é uma p.i.a.c. ergódica para $f$ como no Teorema $\mathrm{C}$ resulta que para qualquer função contínua $h:[0,1] \rightarrow \mathbb{R}$ o conjunto

$$
\Lambda=\left\{x \in[0,1] \text { tal que } \frac{1}{n} \sum_{i=0}^{n-1} h\left(f^{i}(x)\right) \rightarrow \int_{0}^{1} h d \nu \text { quando } n \rightarrow \infty\right\}
$$

tem medida $\nu$ total. Como $f$ é ergódica $[\mathrm{StV}], f^{-1}(\Lambda)=\Lambda$ e $\nu$ é absolutamente contínua com relação à medida de Lebesgue verifica-se também que $|\Lambda|=1$. Neste caso a medida $\nu$ é dita uma medida de Sinai-Ruelle-Bowen (SRB).

Do Lema 3.1 no Capítulo 3 concluímos que os pontos críticos $c$ e $d$ de um polinômio cúbico de Fibonacci estão em um conjunto de Cantor minimal $\Omega$ que por $[\mathrm{StV}]$ tem medida de Lebesgue nula. Neste caso o Teorema $\mathrm{C}$ implica que a bacia de atração de $\Omega$, a saber o conjunto

$$
B(\Omega)=\{x \in[0,1] \text { tal que } \omega(x) \subseteq \Omega\},
$$

tem medida de Lebesgue nula. 


\section{Capítulo 2}

\section{Ferramentas Básicas}

\subsection{Princípios de Koebe e Outros Fatos}

Se $g:[0,1] \rightarrow[0,1]$ é uma aplicação de classe $C^{3}$ podemos definir a sua derivada de Schwarz, $S g$, pela seguinte fórmula:

$$
S g(x)=\frac{g^{\prime \prime \prime}(x)}{g^{\prime}(x)}-\frac{3}{2}\left(\frac{g^{\prime \prime}(x)}{g^{\prime}(x)}\right)^{2},
$$

para todo $x$ que não seja um ponto crítico de $g$. Se $x$ é um ponto crítico definimos $S g(x)=-\infty$.

A derivada de Schwarz desempenha um papel importante em sistemas dinâmicos unidimensionais. Singer provou em [Si] que se $g$ possui derivada de Schwarz negativa (isto é, $S g(x)<0$ para todo $x$ o que abreviamos por $S g<0$ ) então todo ponto periódico atrator de $g$ atrai um ponto crítico ou um dos extremos do seu domínio. Os polinômios $f$ que estamos considerando possuem derivada de Schwarz negativa e como estamos assumindo que os pontos críticos são recorrentes e nãoperiódicos e que os extremos são pontos fixos repulsores, temos que $f$ não possui pontos periódicos atratores.

O conceito de distorção que introduziremos a seguir desempenha um papel central na análise de propriedades métricas.

Definição 2.1. Se gé um difeomorfismo do intervalo $U$ sobre a sua imagem definimos

$$
\operatorname{dist}(g, U)=\sup \left\{\frac{g^{\prime}(x)}{g^{\prime}(y)} \quad \text { onde } \quad x, y \in U\right\}
$$

que chamamos de distorção de $g$ em $U$. 
A derivada de Schwarz também se tornou uma ferramenta útil para o controle de distorção de iteradas de aplicações do intervalo. Os principais fatos nesta direção são os princípios de Koebe enunciados no lema abaixo cuja prova pode ser encontrada em [MSt]. Para enunciá-los introduzimos alguns conceitos. Denotaremos por $|A|$ a medida de Lebesgue de um Boreleano $A$. Se $\sigma>0$ e $U \subset V$ são intervalos tais que para cada componente conexa $L$ de $V \backslash U$ verifica-se que $|L| \geq \sigma|U|$ dizemos que $V$ é uma $\sigma$-vizinhança de $U$ ou que $U$ está $\sigma$-centralizado em $V$.

Lema 2.2. Sejam $U \subset V$ intervalos e $g$ um difeomorfismo de $V$ sobre $g(V)$. Se $S g<0$ e $g(V)$ é uma $\sigma$-vizinhança de $g(U)$ então verifica-se que:

1. Existe $\alpha>0$ tal que $V$ é uma $\alpha$-vizinhança de $U$ e $\alpha$ depende apenas de $\sigma$.

2. $\operatorname{dist}(g, U) \leq\left(\frac{1+\sigma}{\sigma}\right)^{2}$.

As definições a seguir facilitarão a descrição de propriedades de extensões de funções.

Definição 2.3. Uma aplicação real contínua $h$ definida em um intervalo fechado $U$ é dita unimodal se $h(\partial U)$ é um único ponto e existe um ponto $x$ no interior de $U$ tal que $h$ é estritamente monótona em cada uma das componentes de $U \backslash\{x\}$.

Definição 2.4. Sejam $V$ um intervalo e $F=f^{k} u m$ difeomorfismo de $V$ sobre $F(V)$. Se existe um intervalo $\widehat{V} \supset V$ restrito ao qual $f^{k}$ é um difeomorfismo e $f^{k}(\widehat{V})$ é uma $\alpha$-vizinhança de $f^{k}(V)$ dizemos que $F_{\mid V}$ é $\alpha$-extensível. Neste caso denotamos $f_{\mid \hat{V}}^{k}$ por $\widehat{F}$.

- Suponhamos que $f$ e $f^{k+1}$ são aplicações unimodais definidas em um intervalo $U$ e que $f^{k}$ é um difeomorfismo definido em um intervalo $V$ tal que $f(U) \subset V$. Então, se existem intervalos $\widehat{U} \supset U$ e $\widehat{V} \supset V$ tais que $f^{-1}(\partial \widehat{V}) \subset \widehat{U}, f_{\widehat{U}}$ é unimodal e $f_{\mid \widehat{V}}^{k}$ é um difeomorfismo cuja imagem é uma $\alpha$-vizinhança de $f^{k}(V)$ dizemos que a aplicação unimodal $h=f_{\mid U}^{k+1}$ é $\alpha$-extensível. Neste caso denotamos $f_{\mid \widehat{U}}^{k+1}$ por $\widehat{h}$.

O teorema a seguir, cuja demonstração pode ser encontrada em [Ma] e [MSt], assim como o Lema 2.6 serão importantes ferramentas na estimativa de medidas de pré-imagens de intervalos.

Teorema 2.5. Seja $f:[0,1] \rightarrow[0,1]$ uma aplicação de classe $C^{2}$ sem órbitas periódicas atratoras. Seja $U$ um aberto contendo os pontos críticos de $f$. Se todas as órbitas periódicas de $f$ forem hiperbólicas, então existe $C>0$ e $\beta>1$ tal que

$$
\left|D f^{n}(x)\right| \geq C \beta^{n}
$$


se $f^{i}(x) \notin U$ para $0 \leq i \leq n-1$.

Lema 2.6. Seja $f:[0,1] \rightarrow[0,1]$ uma aplicação bimodal de classe $C^{2}$ sem órbitas periódicas atratoras. Sejam $c$ e d seus pontos críticos e sejam $U$ e $V$ intervalos abertos tais que $c \in U, d \in V$ e $f^{j}(\partial U \cup \partial V) \cap(U \cup V)=\emptyset \forall j \geq 0$, com órbita finita. Se para cada $n \geq 1$,

$$
\Lambda_{n}=\left\{x \in[0,1] \backslash\{c, d\} \mid f^{i}(x) \notin U \cup V, 1 \leq i \leq n-1\right\},
$$

então $\left|\Lambda_{n}\right| \rightarrow 0$ exponencialmente quando $n \rightarrow \infty$.

Demonstração: Dado $n \geq 1$, seja $E$ uma componente conexa de $\Lambda_{n}$. Temos $f^{l}(\partial E) \in(\partial U \cup \partial V)$ para algum $l<n$ e $f^{i}(E) \cap(U \cup V)=\emptyset$ para $1 \leq i \leq n-1$ e $f_{\mid E}^{n}$ é monótona. Assim, $f^{n}(\partial E)$ são pontos distintos pertencentes à união das órbitas de $\partial U$ e $\partial V$. Portanto, existe $\eta>0$ tal que $\left|f^{n}(E)\right|>\eta$. Assim, existe $N \in \mathbb{N}$ (que depende de $U$ e de $V$, mas não de $n$ ), tal que $c \in f^{k}(E)$ ou $d \in f^{k}(E)$ para algum $k$ (que podemos escolher o menor possivel) com $n \leq k \leq n+N$. Como $f_{\mid E}^{k}$ é monótona, $f^{k}(E) \supset U$ ou $f^{k}(E) \supset V$. Como $f^{i}(E) \cap(U \cup V)=\emptyset$ para $1 \leq i \leq k-1$, verificamos que existe $K>0$ tal que para $x, y \in E$,

uma vez que

$$
\frac{\left|D f^{k}(x)\right|}{\left|D f^{k}(y)\right|} \leq K
$$

$$
\begin{aligned}
\log \left(\frac{\left|D f^{k}(x)\right|}{\left|D f^{k}(y)\right|}\right) & =\log \left(\frac{\prod_{i=1}^{k}\left|D f\left(f^{k-i}(x)\right)\right|}{\prod_{i=1}^{k}\left|D f\left(f^{k-i}(y)\right)\right|}\right) \\
& =\sum_{i=1}^{k}\left(\log \left(\left|D f\left(f^{k-i}(x)\right)\right|\right)-\log \left(\left|D f\left(f^{k-i}(y)\right)\right|\right)\right) \\
& \leq C \sum_{i=1}^{k}\left|f^{k-i}(x)-f^{k-i}(y)\right| \leq C \sum_{i=1}^{k} \frac{1}{\beta^{i}} \leq C \frac{\beta-1}{\beta}
\end{aligned}
$$

onde $\beta>1$ é a constante dada pelo Teorema 2.5 , e $C>0$ inclui a constante de Lipshitz para $\log (|D f(x)|)$.

Logo, se $U \subset f^{k}(E)$, temos

$$
\frac{|U|}{\left|f^{k}(E)\right|} \leq K \frac{\left|f^{-k}(U) \cap E\right|}{|E|}
$$

e, conseqüentemente $\left|f^{-k}(U) \cap E\right| \geq \alpha|E|$ para algum $\alpha>1$. O mesmo acontece se $V \subset f^{k}(E)$. Então $\left|\Lambda_{n+N}\right| \leq \alpha\left|\Lambda_{n}\right|$ para algum $\alpha<1$ que não depende de $n$ e o lema segue. 


\subsection{Teorema do Folclore}

O teorema abaixo encontra-se demonstrado em [MSt] e é um resultado bastante conhecido sobre a existência de medidas invariantes.

Teorema 2.7. (Folclore) Seja $F: \cup_{j=1}^{\kappa} N_{j} \rightarrow[0,1]$ uma aplicação de Markov de classe $C^{2}$ e hiperbólica em $[0,1]$. Então verificam-se as seguintes propriedades:

1. Existe $K<\infty$ tal que

$$
\frac{\left|D F^{n}(x)\right|}{\left|D F^{n}(y)\right|} \leq K,
$$

para todo $n \geq 0$ e $x, y$ no domínio de um ramo de $F^{n}$.

2. F possui uma medida de probabilidade invariante, ergódica e equivalente à medida de Lebesgue na imagem de F.

Demonstração: Para demonstrarmos a primeira afirmação observamos que dados $x, y \in N_{j}$ para algum $j>0$,

$$
\begin{aligned}
\log \left(\frac{\left|D F^{n}(x)\right|}{\left|D F^{n}(y)\right|}\right) & =\log \left(\frac{\prod_{i=1}^{n}\left|D F\left(F^{n-i}(x)\right)\right|}{\prod_{i=1}^{n}\left|D F\left(F^{n-i}(y)\right)\right|}\right) \\
& =\sum_{i=1}^{n}\left(\log \left(\left|D F\left(F^{n-i}(x)\right)\right|\right)-\log \left(\left|D F\left(F^{n-i}(y)\right)\right|\right)\right) \\
& \leq C \sum_{i=1}^{n}\left|F^{n-i}(x)-F^{n-i}(y)\right| \leq C \sum_{i=1}^{n} \frac{1}{a^{i}} \leq C \frac{a-1}{a}
\end{aligned}
$$

onde $a>1$ é a constante dada pela definição 1.2 , e $C>0$ é a constante de Lipshitz para $\log (|D F(x)|)$. Para demonstrarmos a segunda afirmação, dado $i \in$ $\mathbb{N}$, tomamos $\lambda_{i}$ a medida de probabilidade definida nos borelianos de $[0,1]$ por $\lambda_{i}(B)=\left|F^{-i}(B)\right|$. O espaço de medidas de probabilidade em $[0,1]$ munido da topologia fraca é compacto. Portanto, a seqüência

$$
\mu_{n}:=\frac{1}{n} \sum_{i=0}^{n-1} \lambda_{i}
$$

possui uma subseqüência convergente, ou seja, existe uma medida de probabilidade $\mu$ e uma subsequência $\mu_{n_{k}}$ tal que $\mu_{n_{k}} \rightarrow \mu$ quando $n_{k} \rightarrow \infty$. Além disso, $\mu$ é uma medida $F$-invariante. De fato, se $B$ é um boreliano de $N$,

$$
\mu\left(F^{-1}(B)\right)=\lim _{k \rightarrow \infty} \frac{1}{n_{k}} \sum_{i=0}^{n_{k}-1}\left|F^{-i-1}(B)\right|
$$




$$
\begin{aligned}
& =\lim _{k \rightarrow \infty}\left(\frac{1}{n_{k}} \sum_{i=0}^{n_{k}-1}\left|F^{-i}(B)\right|+\frac{1}{n_{k}}\left|F^{-n_{k}}(B)\right|-\frac{1}{n_{k}}|B|\right) \\
& =\lim _{k \rightarrow \infty} \frac{1}{n_{k}} \sum_{i=0}^{n_{k}-1}\left|F^{-i}(B)\right|=\mu(B)
\end{aligned}
$$

Esta medida $\mu$ é absolutamente contínua em relação à medida de Lebesgue. Para mostrarmos isso, vamos considerar a partição $\mathcal{P}$ de $[0,1]$ formada por $\cup N_{j}$ e a partição imagem $F(\mathcal{P})$. Seja $E$ contido na imagem de $F$ um subconjunto mensurável e $E_{i}=E \cap F\left(N_{i}\right)$. Observamos que para $n \geq 1$ a partição $F^{-n}(\mathcal{P})$ refina a partição $F^{-n+1}(\mathcal{P})$. Fixamos $i$ e dado $n>0$, seja $A$ um átomo de $F^{-n+1}(\mathcal{P})$ o qual é aplicado difeomorficamente em $F\left(N_{i}\right)$ por $F^{n}$. Seja $L \subset A$ tal que $F^{n}(L)=E_{i}$. Então

$$
\left|E_{i}\right|=\int_{L}\left|D F^{n}(x)\right| d x
$$

Como $F^{n}: A \rightarrow F\left(N_{i}\right)$ é um difeomorfismo com distorção limitada por $K$ obtemos

$$
\frac{1}{K} \frac{\left|F\left(N_{i}\right)\right|}{|A|} \int_{L} d x<\left|E_{i}\right|<K \frac{\left|F\left(N_{i}\right)\right|}{|A|} \int_{L} d x
$$

e, conseqüentemente,

$$
\frac{1}{K} \frac{\left|E_{i}\right|}{\left|F\left(N_{i}\right)\right|}<\frac{\left|F^{-n}\left(E_{i}\right) \cap A\right|}{|A|}<K \frac{\left|E_{i}\right|}{\left|F\left(N_{i}\right)\right|} .
$$

Somando para todos os A's, temos

$$
\frac{1}{K} \frac{\left|E_{i}\right|}{\left|F\left(N_{i}\right)\right|}<\frac{\left|F^{-n}\left(E_{i}\right)\right|}{\left|F^{-n}\left(F\left(N_{i}\right)\right)\right|}<K \frac{\left|E_{i}\right|}{\left|F\left(N_{i}\right)\right|},
$$

ou seja,

$$
\frac{1}{K}\left|E_{i}\right| \frac{\left|F^{-n}\left(F\left(N_{i}\right)\right)\right|}{\left|F\left(N_{i}\right)\right|}<\left|F^{-n}\left(E_{i}\right)\right|<K\left|E_{i}\right| \frac{\left|F^{-n}\left(F\left(N_{i}\right)\right)\right|}{\left|F\left(N_{i}\right)\right|} .
$$

Lembramos que a partição imagem é finita. Como $\left|F^{-n}([0,1])\right|=|[0,1]|$, existe um conjunto não-vazio $\mathcal{I}$ tal que para todo $i \in \mathcal{I}, \mu\left(F\left(N_{i}\right)\right)>0$. Sejam

$$
M_{1}=\min _{i \in \mathcal{I}} \frac{\mu\left(F\left(N_{i}\right)\right)}{\left|F\left(N_{i}\right)\right|} \quad \text { e } \quad M_{2}=\max _{i \in \mathcal{I}} \frac{\mu\left(F\left(N_{i}\right)\right)}{\left|F\left(N_{i}\right)\right|} .
$$

Então, para cada $i \in \mathcal{I}$,

$$
M_{1} \frac{1}{K}\left|E_{i}\right|<\mu\left(E_{i}\right)<M_{2} K\left|E_{i}\right|
$$


e, usando novamente que a partição imagem é finita, obtemos

$$
M_{1} \frac{1}{K}|E|<\mu(E)<M_{2} K|E|
$$

para cada $E$ mensurável. Logo $\mu$ é equivalente à medida de Lebesgue na imagem de $F$ e é finita.

A ergodicidade de $\mu$ é uma consequência imediata do fato de que os ramos de iteradas de $F$ têm distorção limitada o que nos permite passar de escalas arbitrariamente pequenas para um dos intervalos na partição imagem de $F$. Isto garante que todo conjunto invariante com medida de Lebesgue positiva (o suporte de uma p.i.a.c. por exemplo) tem de fato medida de Lebesgue total na imagem de $F$.

\subsection{Média Temporal}

A partir da p.i.a.c. existente para a aplicação de Markov $F$ induzida por $f$ poderemos contruir uma medida invariante para esta última. Esta medida será uma p.i.a.c. caso tenhamos a finitude da média temporal do lema abaixo.

Lema 2.8. Suponha que $f:[0,1] \rightarrow[0,1]$ induz uma aplicação de Markov $F$ : $\cup_{j=1}^{\kappa} N_{j} \rightarrow[0,1], \kappa \leq \infty \operatorname{com} F_{\mid N_{j}}=f_{\mid N_{j}}^{n_{j}}$. Se $\mu$ é uma p.i.a.c. de $F$ e se

$$
\sum_{j=1}^{\kappa} n_{j} \mu\left(N_{j}\right)<\infty
$$

então $f$ possui uma p.i.a.c.

Observação 2.9. Observamos que se

$$
\sum_{j=1}^{\kappa} n_{j}\left|N_{j}\right|<\infty
$$

e existe constante $K<\infty$ tal que $\mu\left(N_{j}\right)<K\left|N_{j}\right|$ resulta que 2.2 é verdadeira. Isso é o que ocorre de fato se $\mu$ é a medida dada pelo Teorema 2.7.

Demonstração: Denotamos por $\mu_{j}$ a restrição de $\mu$ a $N_{j}$, ou seja, $\mu_{j}(A)=$ $\mu\left(A \cap N_{j}\right)$, para cada $A$ mensurável. Assim, $\mu=\sum_{j=1}^{\kappa} \mu_{j}$. Vamos definir uma nova medida $\nu$ pela fórmula

$$
\nu(A)=\sum_{j=1}^{k} \sum_{i=0}^{n_{j}-1} \mu_{j}\left(f^{-i}(A)\right.
$$


para cada $A$ mensurável. Pela definição, $\nu$ é absolutamente contínua em relação à medida de Lebesgue uma vez que assim é $\mu$. Falta demonstrar que $\nu$ é $f$-invariante. De fato, para cada $A$ mensurável,

$$
\mu\left(f^{-1}(A)\right)=\sum_{j=1}^{k} \sum_{i=0}^{n_{j}-1} \mu_{j}\left(f^{-i-1}(A)\right)=\sum_{j=1}^{k}\left(\sum_{i=0}^{n_{j}-1} \mu_{j}\left(f^{-i}(A)\right)+\mu_{j}\left(f^{-n_{j}}(A)\right)\right)
$$

Tem-se que

$$
\begin{gathered}
\mu_{j}\left(f^{-n_{j}}(A)\right)=\mu\left(f^{-n_{j}}(A) \cap N_{j}\right)=\mu\left(F^{-1}(A) \cap N_{j}\right), \\
\mu\left(F^{-1}(A)\right)=\sum_{j=1}^{\kappa} \mu\left(F^{-1}(A) \cap N_{j}\right) \quad \text { e } \mu(A)=\sum_{j=1}^{\kappa} \mu\left(A \cap N_{j}\right) .
\end{gathered}
$$

Pela $F$-invariância de $\mu$, temos que

$$
\sum_{j=1}^{\kappa} \mu_{j}\left(f^{-n_{j}}(A)\right)=\sum_{j=1}^{\kappa} \mu_{j}(A)
$$

Portanto, $\nu$ é $f$-invariante. Como

$$
\nu([0,1])=\sum_{j=1}^{k} \sum_{i=0}^{n_{j}-1} \mu_{j}\left(f^{-i}([0,1])\right)=\sum_{j=1}^{\kappa} n_{j} \mu\left(N_{j}\right)
$$

resulta que se vale 2.2 então $\nu([0,1])$ é finita.

O teorema abaixo permite provar a existência de uma p.i.a.c. para $f$ usando apenas uma condição de somabilidade nas órbitas críticas e está demonstrado em [BSt].

Teorema 2.10. Seja $f:[0,1] \rightarrow[0,1]$ uma aplicação multimodal de classe $C^{3}$ com derivada de Schwarz negativa e um número finito de pontos críticos com ordem máxima finita l. Se para cada ponto crítico c vale

$$
\sum_{k=1}^{\infty}\left(\left|D f^{k}(f(c))\right|\right)^{-1 / l}<\infty
$$

então $f$ admite uma medida de probabilidade $\nu$, invariante e absolutamente contínua em realção à medida de Lebesgue e $\mu \in L^{\tau}$ para qualquer $\tau<l /(l-1)$. 


\section{Capítulo 3}

\section{Polinômios de Fibonacci}

\subsection{Existência de Polinômios Fibonacci}

Começamos este capítulo com o lema abaixo demonstrado em [SV] que garante a existência de uma importante classe de polinômios cúbicos bimodais a qual contém os de Fibonacci.

Lema 3.1. Existem parâmetros $a, b$ tais que o polinômio cúbico $f=f_{a b}=a x^{3}+$ $b x^{2}+(1-a-b) x$ é bimodal e cuja seqüência de primeiros retornos $\phi_{n}$, para $n \geq 1$, satisfaz as seguintes propriedades:

1. Existem duas componentes conexas do domínio de $\phi_{n}$ que denotamos por $U_{n+1}^{c}$ e $U_{n+1}^{d}$ e que contêm os valores críticos $\phi_{n}(c)$ e $\phi_{n}(d)$, respectivamente.

2. As imagens dos ramos críticos $\phi_{n \mid \delta_{n+1}} e \phi_{n \mid \gamma_{n+1}}$ estão ambas contidas em $\delta_{n}$ ou ambas em $\gamma_{n}$. Neste primeiro caso tem-se que $\delta_{n+1} \subset \phi_{n}\left(\gamma_{n+1}\right)$ e no segundo caso tem-se que $\gamma_{n+1} \subset \phi_{n}\left(\delta_{n+1}\right)$

3. Os ramos $\phi_{n \mid U_{n+1}^{c}} e \phi_{n \mid U_{n+1}^{d}}$ são monótonos e suas imagens coincidem e, no caso em que $\phi_{n}(d) \in \delta_{n}$ e $\phi_{n}(c) \in \delta_{n}$, são iguais a $\gamma_{n}$. No caso em que $\phi_{n}(d) \in \gamma_{n}$ e $\phi_{n}(c) \in \gamma_{n}$, são iguais a $\delta_{n}$.

Existem vários polinômios $f_{a b}$ satisfazendo as propriedades descritas no Lema 3.1. Por construção estes polinômios não apresentam retornos centrais mas pode ocorrer uma forte recorrência dos pontos críticos que é o caso por exemplo dos polinômos de Fibonacci cuja seqüência de aplicações de primeiro retorno $\left\{\phi_{n}\right\}$ satisfaz $\phi_{0}(c)=f(c), \phi_{0}(d)=f^{2}(d), \phi_{1}(c)=f^{2}(c), \quad \phi_{1}(d)=f^{2}(d)$, $\phi_{n+1}(c)=\phi_{n-1}\left(\phi_{n}(c)\right)$ e $\phi_{n+1}(d)=\phi_{n-1}\left(\phi_{n}(d)\right)$. Então temos que já na segunda 
iterada os pontos críticos retornam a $\delta_{n+1} \cup \gamma_{n+1}$ e podemos dizer que estamos o mais próximo possível da situação em que retornos centrais ocorrem. Por isto, para efeitos da existência de uma p.i.a.c. a partir do decaimento exponencial de geometria, esta situação é a mais delicada. Sendo assim esperamos que as outras situações possam ser tratadas pelos mesmos métodos e de maneira mais fácil.

Demonstração do Lema 3.1 Esta prova será baseada no seguinte fato.

Fato: Seja $g:[0,1] \rightarrow[0,1]$ uma aplicação bi-modal contínua tal que $g(0)=0$, $g(1)=1$ e os seus pontos extremos locais em $(0,1)$ (que chamaremos de pontos críticos) não são periódicos. Então existe uma aplicação $f_{a b}$ da família cúbica acima tal que $g$ é semi-conjugada a $f_{a b}$. A semi-conjugação pode deixar de ser injetiva apenas nos intervalos errantes de $g$ ou em intervalos que são assintóticos a pontos periódicos atratores de $g$. Veja [MSt] para uma prova deste fato.

Obteremos então uma aplicação bimodal contínua $g$ satisfazendo o Lema 3.1 como limite de uma seqüência $g_{n}$ de aplicações bimodais construídas indutivamente.

Indução (primeiro passo): Este passo de indução está ilustrado nas figuras 3.1 e 3.3. Seja $g_{0}:[0,1] \rightarrow[0,1]$ uma aplicação bimodal contínua com pontos críticos $c$ e $d$ e tal que $g_{0}$ é estritamente crescente em $[0, c]$ e possui um único ponto fixo $p$ em $(0,1)$. Observamos que $[0, p]=\bigcup_{i=0}^{\infty} U_{i}$ e $[p, 1]=\bigcup_{i=0}^{\infty} V_{i}$ onde $U_{i}$ e $V_{i}$ são intervalos tais que $g_{0}^{i}\left(U_{i}\right)=\delta_{0}=\left(p_{c}, p\right)$ e $g_{0}^{i}\left(V_{i}\right)=\gamma_{0}=\left(p, p_{d}\right)$. Denotamos por $p_{c} \in(0, p)$ e $p_{d} \in(p, 1)$ os únicos pontos tais que $f\left(p_{c}\right)=f\left(p_{d}\right)=f(p)=p$. Então podemos escolher $g_{0}$ de modo que $g_{0}(c) \in V_{j} \operatorname{com} j \geq 0$ e $g_{0}(d) \in U_{k} \operatorname{com} k \geq 1$. Observamos que no caso $j=0$ ocorre que $\delta_{0}=\delta_{1}$ e $\gamma_{1}=\gamma_{2}$. Então, se $\phi_{0}$ é a aplicação de primeiro retorno a $\delta_{0} \cup \gamma_{0}$ resulta que $\phi_{0}\left(\delta_{1}\right) \subset \gamma_{0}$ e $\phi_{0}\left(\gamma_{1}\right) \subset \delta_{0}$, onde $\delta_{1} \ni c$ e $\gamma_{1} \ni d$ são os domínios críticos de $\phi_{0}$.

Alterando $g_{0}$ apenas em $\delta_{1} \cup \gamma_{1}$ obtemos uma aplicação bimodal $g_{1}$ cujos valores críticos estão posicionados de tal forma que $\phi_{1}$, a aplicação de primeiro retorno a $\delta_{1} \cup \gamma_{1}$ correspondente, satisfaça as afirmações 1-3 do Lema $3.1 \mathrm{com}$ ambos $\phi_{1}(c)$ e $\phi_{1}(d)$ pertencentes a $\delta_{1}$.

Observamos que, desde que $\phi_{1}$ é a aplicação de primeiro retorno de $\delta_{1} \cup \gamma_{1}$, podemos modificar $g_{1}$ em $\delta_{2} \cup \gamma_{2}$ de tal maneira que todos os ramos monótonos de $\phi_{1}$ permaneçam inalterados e os valores críticos $\phi_{1}(c)$ e $\phi_{1}(d)$ sejam movidos independentemente para posições pré-designadas em $\delta_{1}$.

Indução (passo geral): Este passo de indução está ilustrado na figura 3.2. Suponhamos que temos uma aplicação bi-modal $g_{n}$ escolhida de tal maneira que $\phi_{n}$ satisfaz o Lema 3.1. Assumimos que a imagem dos ramos críticos de $\phi_{n}$ estão contidas em $\delta_{n}$ (o caso em que estão contidas em $\gamma_{n}$ é análogo). Temos também que modificando $g_{n}$ somente em $\delta_{n+1} \cup \gamma_{n+1}$ os ramos monótonos de $\phi_{n}$ permanecem 

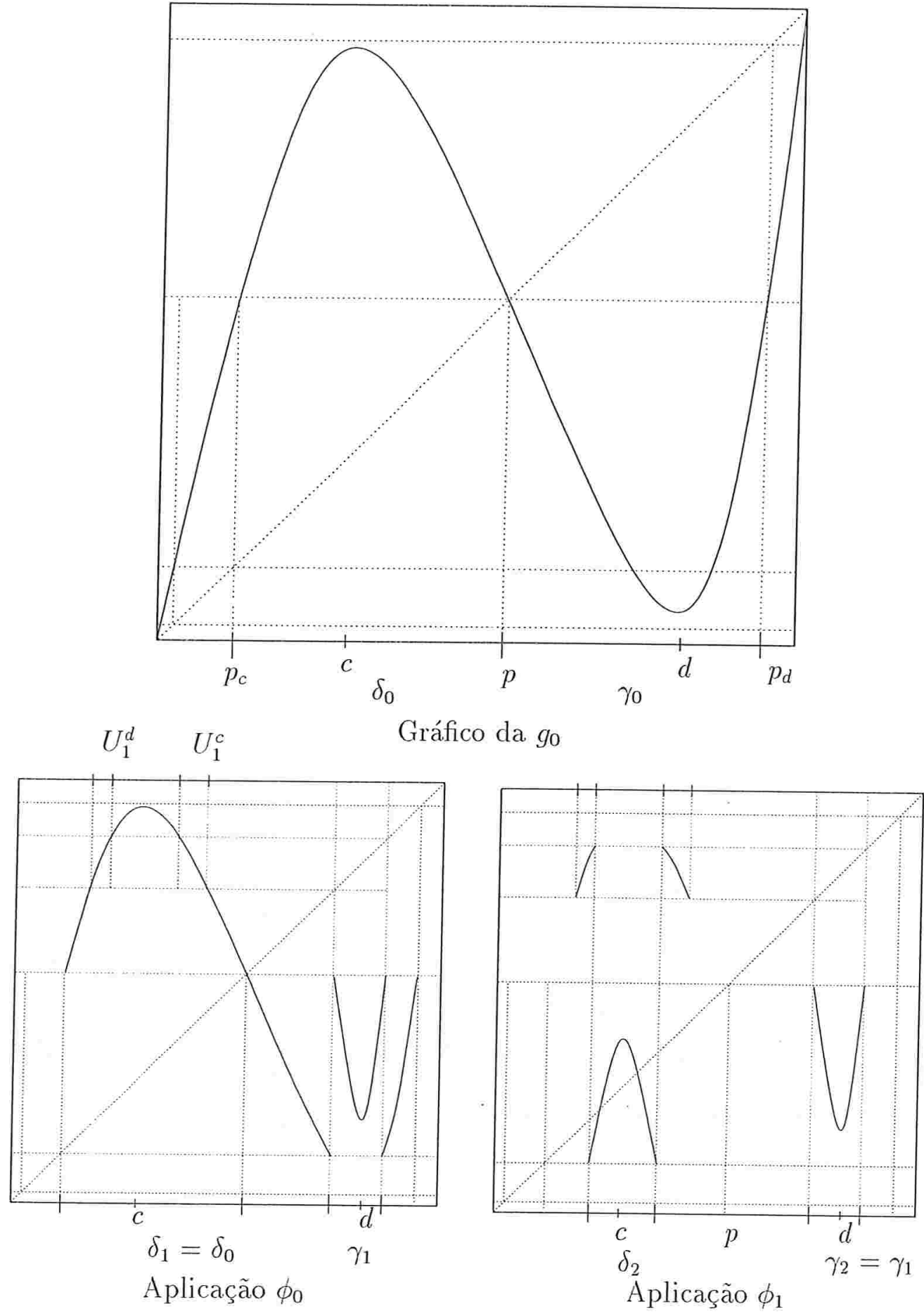

Figura 3.1: Aplicações de primeiro retorno

inalterados e os valores críticos $\phi_{n}(c)$ e $\phi_{n}(d)$ podem ser movidos livremente para 


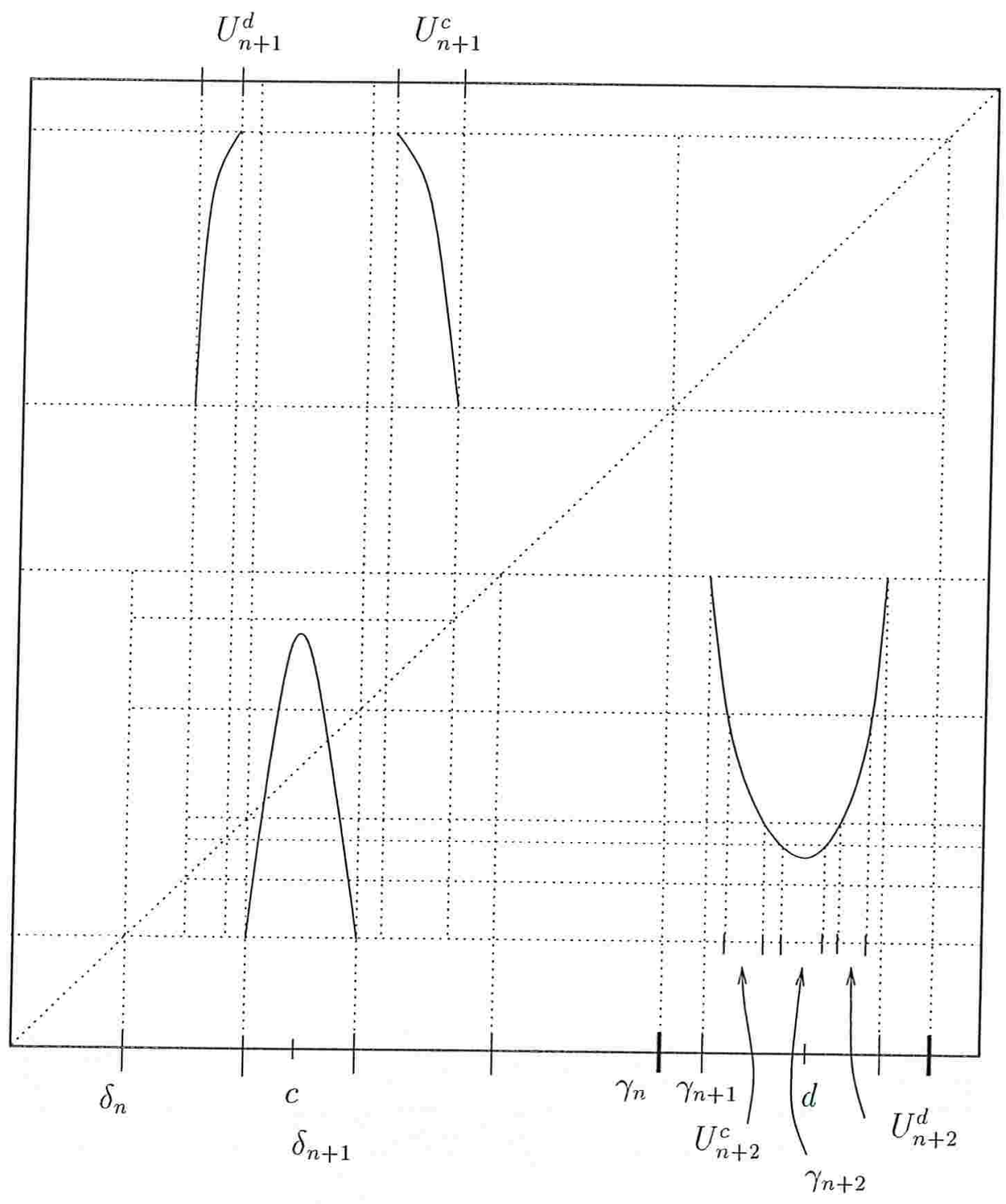

Figura 3.2: A aplicação $\phi_{n}$.

quaisquer posições pré-designadas em $\delta_{n}$. Na verdade precisamos apenas mover estes valores críticos livremente em $U_{n+1}^{c}$ e $U_{n+1}^{d}$, respectivamente.

A aplicação $\phi_{n+1}$ é o primeiro retorno a $\delta_{n+1} \cup \gamma_{n+1}$. Os domínios $U_{n+2}^{c}$ e $U_{n+2}^{d}$ de $\phi_{n+1}$ são os dois intervalos cuja união é $\phi_{n \mid \gamma_{n+1}}^{-1}\left(\delta_{n+1}\right)$. Nestes domínios $\phi_{n+1}$ é monótona e igual a $\phi_{n}$. As suas imagens coincidem e são iguais a $\delta_{n+1}$. Como $\phi_{n}(c)$ e $\phi_{n}(d)$ podem variar livremente em $U_{n+1}^{c}$ e $U_{n+1}^{d}$, respectivamente, modificamos $g_{n}$ em $\delta_{n+1} \cup \gamma_{n+1}$ e obtemos uma aplicação bimodal $g_{n+1}$ de tal modo que $\phi_{n}(c) \in \phi_{n \mid U_{n+1}^{c}}^{-1}\left(\gamma_{n+1}\right)$ e $\phi_{n}(d) \in \phi_{n \mid U_{n+1}^{d}}^{-1}\left(\gamma_{n+1}\right)$. Resulta então que $\phi_{n+1}=\phi_{n}^{2}$ em $\delta_{n+2} \cup \gamma_{n+2}$. As imagens de $\phi_{n+1 \mid \delta_{n+2}}$ e de $\phi_{n+1 \mid \gamma_{n+2}}$ estão ambas contidas em 


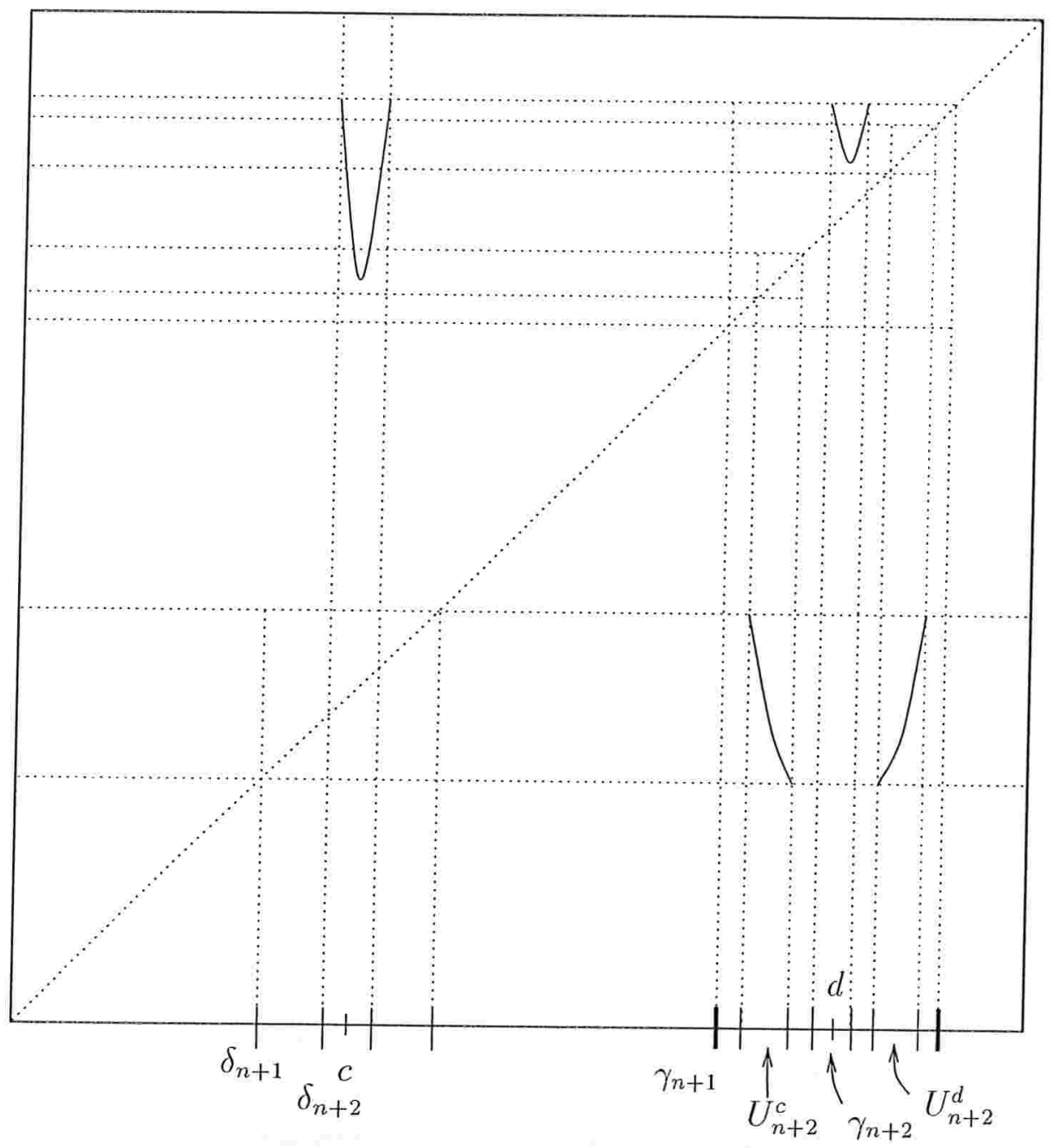

Figura 3.3: A aplicação $\phi_{n+1}$.

$\gamma_{n+1}$. Finalmente observamos que modificando $g_{n+1}$ apenas em $\delta_{n+2} \cup \gamma_{n+2}$ os ramos monótonos de $\phi_{n+1}$ permanecem inalterados e os valores críticos $\phi_{n+1}(c)$ e $\phi_{n+1}(d)$ podem ser movidos livremente para quaisquer posições pré-designadas em $U_{n+2}^{c}$ e $U_{n+2}^{d}$. Além disto $\phi_{n+1}$ satisfaz as afirmações de 1-3 do Lema 3.1.

Podemos implementar esta construção de modo que $\cap_{n=0}^{\infty} \delta_{n}=\{c\}$ e $\cap_{n=0}^{\infty} \gamma_{n}=$ $\{d\}$. Assim, a seqüência $g_{n}$ converge, quando $n \rightarrow \infty$, para uma aplicação bimodal contínua $g$ a qual satisfaz o Lema 3.1. Então o polinômio $f_{a b}$ ao qual $g$ é semi-conjugada satisfaz as afirmações do lema como queremos.

Em [SV], demonstra-se que para os polinômios satisfazendo o Lema 3.1 ocorre decaimento de geometria, definindo, porém, os intervalos centrais que contém $c$ e $d$ como domínios de primeiro retorno ao intervalo que contém $c$ e não à união dos 
dois intervalos como definimos aqui. Contudo, daquela demonstração, podemos concluir que se os intervalos centrais $\delta_{n}$ e $\gamma_{n}$ forem tomados como definidos lá, então $\frac{\left|U_{n+1}^{c}\right|}{\left|\delta_{n}\right|}$ e $\frac{\left|U_{n+1}^{d}\right|}{\left|\delta_{n}\right|}$ decrescerão exponecialmente, assim como $\frac{\left|U_{n+1}^{c}\right|}{\left|\delta_{n}\right|}$ e $\frac{\left|U_{n+1}^{d}\right|}{\left|\delta_{n}\right|}$. Além disso, para $\phi_{n}$ restrita aos intervalos centrais como definida em [SV], usando o decaimento de geometria (e os mesmos argumentos que usamos na demostração do Corolário 4.6), podemos mostrar que existe uma constante $K>0$ tal que

$$
K|x-c| \leq\left|D \phi_{n}(x)\right| \leq K|x-c|
$$

se $x$ pertence à componente conexa do domínio de $\phi_{n}$ que contém $c$ e

$$
K|x-d| \leq\left|D \phi_{n}(x)\right| \leq K|x-d|
$$

se $x$ pertence à componente conexa do domínio de $\phi_{n}$ que contém $d$. Com isso, podemos demonstrar que para os intervalos $\delta_{n}$ e $\gamma_{n}$ construídos aqui temos que as razões

$$
\frac{\left|\delta_{n+1}\right|}{\left|\delta_{n}\right|} \text { e. } \frac{\left|\gamma_{n+1}\right|}{\left|\gamma_{n}\right|}
$$

tendem a zero exponencialmente quando $n \rightarrow \infty$.

\subsection{Fibonacci Cúbico, Propriedades Topológicas}

Lema 3.2. Quaisquer dois polinômios cúbicos de Fibonacci são topologicamente conjugados.

Demonstração: É suficiente verificarmos que duas aplicações bimodais Fibonacci possuem valores críticos com as mesmas sequências de kneading (veja [MSt]). Para isto precisamos apenas provar que uma aplicação bimodal Fibonacci determina uma sequência de aplicações de primeiro retorno que satisfaz o Lema $3.1 \mathrm{com}$ $\phi_{1}(c)=f^{2}(c), \phi_{1}(d)=f^{2}(d), \phi_{2}(c)=f^{3}(c)$ e $\phi_{2}(d)=f^{3}(d)$. Do Lema 3.1 seguirá que $\phi_{n+1}=\phi_{n-1} \circ \phi_{n}$ e, portanto, $\phi_{n}(c)=f^{s_{n}}(c)$ e $\phi_{n}(d)=f^{s_{n}}(d)$ para todo $n \geq 2$.

Usaremos indução. Inicialmente temos que $\phi_{0}(c)=f(c)$ e $\phi_{0}(d)=f(d)$ ou $\phi_{0}(c)=f(c)$ e $\phi_{0}(d)=f^{2}(d)$, (ou permutando os pontos críticos $\phi_{0}(c)=f^{2}(c)$ e $\left.\phi_{0}(d)=f(d)\right)$ ou, ainda, $\phi_{0}(c)=f^{2}(c)$ e $\phi_{0}(d)=f^{2}(d)$. Neste último caso para que $\phi_{1}(c)=f^{2}(c)$ e $\phi_{1}(d)=f^{2}(d)$ como requerido teríamos de ter $\phi_{0}(c)$ e $\phi_{0}(d)$ com retornos centrais. Mas isto implicaria que $\phi_{2}(c)=f^{4}(c)$ e $\phi_{2}(d)=f^{4}(d)$ e portanto a aplicação não seria Fibonacci. No primeiro caso, teríamos $f$ renormalizável, $\operatorname{com} \phi_{2}(c)=f^{4}(c)$ e $\phi_{2}(d)=f^{4}(d)$ e novamente não teríamos uma aplicação de 
Fibonacci. Podemos assumir então que $\phi_{0}(c)=f(c)$ e $\phi_{0}(d)=f^{2}(d)$. Resulta que $\delta_{1}=\delta_{0}, \gamma_{2}=\gamma_{1}$ e $\phi_{1 \mid \gamma_{2}}=\phi_{0 \mid \gamma_{1}}$. Devemos ter $\phi_{0}\left(\delta_{1}\right) \supset \gamma_{2}$, caso contrário, teríamos $\delta_{2}=\delta_{1}, \gamma_{3}=\gamma_{2}$ e $\phi_{3}(d)=f^{2}(d)$, contradizendo o fato de que $f$ é um polinômio de Fibonacci. Assim, temos que $\phi_{1}(c)=f^{2}(c), \phi_{1}(d)=f^{2}(d)$ e $\phi_{1}$ satisfaz o Lema 3.1. Então $\phi_{2}(c)=f^{3}(c)$ e $\phi_{2}(d)=f^{3}(d)$. Assumimos que para $k \leq n, \phi_{k}(c)=f^{s_{k}}(c), \phi_{k}(d)=f^{s_{k}}(d)$ e $\phi_{k}$ satisfaz o Lema 3.1. Pela propriedade de Fibonacci verificamos que $\phi_{n+1}=\phi_{n-1} \circ \phi_{n}$ e $\phi_{n+1}$ satisfaz o Lema 3.1 e com isso concluímos a demosntração.

Lema 3.3. Seja $f$ um polinômio cúbico bimodal de Fibonacci. Seus pontos críticos, c e d, estão contidos num conjunto de Cantor minimal.

Demonstração: Para cada $n \geq 1$, definimos

$\Omega_{n}:=\left(\bigcup_{i=0}^{s_{n+1}-1} f^{i}\left(\delta_{n+1}\right)\right) \cup\left(\bigcup_{j=0}^{s_{n}-1} f^{j}\left(U_{n+1}^{c}\right)\right) \cup\left(\bigcup_{k=0}^{s_{n+1}-1} f^{k}\left(\gamma_{n+1}\right)\right) \cup\left(\bigcup_{l=0}^{s_{n}-1} f^{l}\left(U_{n+1}^{d}\right)\right)$

onde $U_{n+1}^{d}, \gamma_{n+1}, U_{n+1}^{c}$ e $\delta_{n+1}$ são os intervalos definidos na demonstração do Lema 3.1. Observamos que para cada $n \geq 1, \Omega_{n}$ contém orb ${ }^{+}(c) \cup \operatorname{orb}^{+}(d)$. De fato, para $0 \leq i \leq s_{n+1}-1$, temos $f^{i}(c) \in \bigcup_{i=0}^{s_{n+1}-1} f^{i}\left(\delta_{n+1}\right)$. Então observamos que

- $f^{s_{n+1}}\left(\delta_{n+2}\right) \subset U_{n+1}^{c}$ e $f^{s_{n+1}}\left(\gamma_{n+2}\right) \subset U_{n+1}^{d}$;

- Para $l \geq 2, f^{s_{n+l}}\left(\delta_{n+l}\right) \cup f^{s_{n+l}}\left(\gamma_{n+l}\right) \subset \gamma_{n+l-1} \cup \delta_{n+l-1}$ e $f^{s_{n+l+1}}(c) \in \gamma_{n+l} \cup \delta_{n+l}$.

Com isso, usando que $s_{n+1}=s_{n}+s_{n-1}$, temos que orb $b^{+}(c) \subset \Omega_{n}$ para todo $n \geq 1$.

De maneira similar, mostramos o mesmo sobre a $\operatorname{orb}^{+}(d)$.

Para cada $n \geq 1$, podemos mostrar também que $\Omega_{n+1} \subset \Omega_{n}$, pois

- $\delta_{n+2} \subset \delta_{n+1}$ e $f^{s_{n+1}}\left(\delta_{n+2}\right) \subset U_{n+1}^{c}$, garantindo

$$
\bigcup_{i=0}^{s_{n+2}-1} f^{i}\left(\delta_{n+2}\right) \subset\left(\bigcup_{i=0}^{s_{n+1}-1} f^{i}\left(\delta_{n+1}\right)\right) \cup\left(\bigcup_{j=0}^{s_{n}-1} f^{j}\left(U_{n+1}^{c}\right)\right) .
$$

- $U_{n+2}^{c} \cup U_{n+2}^{d} \subset \delta_{n+1} \cup \gamma_{n+1}$ e portanto

$$
\left(\bigcup_{j=0}^{s_{n+1}-1} f^{j}\left(U_{n+2}^{c}\right)\right) \cup\left(\bigcup_{l=0}^{s_{n+1}-1} f^{l}\left(U_{n+2}^{d}\right)\right) \subset\left(\bigcup_{i=0}^{s_{n+1}-1} f^{i}\left(\delta_{n+1}\right)\right) \cup\left(\bigcup_{k=0}^{s_{n+1}-1} f^{k}\left(\gamma_{n+1}\right)\right) .
$$


- $\gamma_{n+2} \subset \gamma_{n+1}$ e $f^{s_{n+1}}\left(\gamma_{n+2}\right) \subset U_{n+1}^{d}$, garantindo

$$
\bigcup_{i=0}^{s_{n+2}-1} f^{i}\left(\gamma_{n+2}\right) \subset\left(\bigcup_{i=0}^{s_{n+1}-1} f^{i}\left(\gamma_{n+1}\right)\right) \cup\left(\bigcup_{j=0}^{s_{n}-1} f^{j}\left(U_{n+1}^{d}\right)\right) \text {. }
$$

Observamos que com isso podemos concluir também que dado um intervalo $L$ de $\Omega_{n+1}$ e um intervalo $V$ de $\Omega_{n}$ existe $j \in \mathbb{N}$ tal que $f^{j}(L) \subset V$.

Verificamos ainda que se $m_{n}=\max \left\{|V| \mid V\right.$ é uma componente conexa de $\left.\Omega_{n}\right\}$, então $m_{n} \rightarrow 0$ quando $n \rightarrow \infty$.

Seja

$$
\Omega=\bigcap_{n=1}^{\infty} \Omega_{n}
$$

Temos que $\Omega$ é um conjunto de Cantor e $\operatorname{orb}^{+}(c) \cup \operatorname{orb}^{+}(d) \subset \Omega$. Além disso, dados $\varepsilon>0$ e $x, y \in \Omega$, podemos escolher $n>0$ de modo que os intervalos de $\Omega_{n}$ tenham comprimento inferior a $\varepsilon$. Seja $V \subset \Omega_{n}$ o intervalo que contém $y$. Seja $L$ o intervalo em $\Omega_{n+1}$ que contém $x$. Como existe $j>0$ tal que $f^{j}(L) \subset V$, temos $f^{j}(x) \in(y-\varepsilon, y+\varepsilon)$. Assim, verificamos que dado $x \in \Omega, \operatorname{orb}^{+}(x)$ é densa em $\Omega$, ou seja, $\Omega$ é um conjunto minimal. 


\section{Capítulo 4}

\section{Hiperbolicidade Induzida}

Neste capítulo provaremos o Teorema A e o Teorema B. Fixemos um polinômio cúbico bimodal $f:[0,1] \rightarrow[0,1]$ pertencente à família $f_{a b}(x)=a x^{3}+b x^{2}+(1-$ $a-b) x$ que exiba a combinatória de Fibonacci. Construiremos por indução uma partição módulo zero (ou seja, módulo um conjunto com medida de Lebesgue nula) de $[0,1]$. Os elementos desta partição que denotaremos por $\xi$ serão intervalos nos quais definiremos uma aplicação de Markov $F$ induzida por $f$. Posteriormente provaremos que, para algum $N \geq 1, F^{N}$ é hiperbólica e possui uma p.i.a.c. Determinaremos a aplicação de Markov $F$ ao construirmos a partição $\xi$.

\subsection{Fibonacci Induz Markov}

Inicialmente observamos que no complementar de $\delta_{0}=\left(p_{c}, p\right) \cup \gamma_{0}=\left(p, p_{d}\right)$, podemos tomar pré-imagens disjuntas destes intervalos por iteradas de $f$ e assim temos uma coleção enumerável de intervalos nos quais está definida uma aplicação monótona, dada pela restição de uma iterada de $f$, com imagem igual a $\delta_{1}=\delta_{0}$ ou a $\gamma_{1}$ (veja Figura 3.1). Denotamos cada um destes domínios por $\Delta$ e em cada um deles, definimos um ramo de $F_{1}:=f_{\mid \Delta}^{n_{\Delta}}: \Delta \rightarrow[0,1]$. Em $\delta_{0} \cup \gamma_{0}$, tomamos $\phi_{0}$, a aplicação de primeiro retorno a $\delta_{0} \cup \gamma_{0}$. O domínio de $\phi_{0}$ é uma união finita de intervalos abertos dentre os quais existe um, denotado por $\delta_{1}$, que contém o ponto crítico $c$ e outro, denotado por $\gamma_{1}$, que contém o ponto crítico $d$. Aqueles que não contêm pontos críticos serão denotados também por $\Delta$.

Obtemos assim uma primeira partição de $[0,1]$ :

$$
\xi_{1}:[0,1]=(\cup \Delta) \cup \delta_{1} \cup \gamma_{1}
$$

Em cada intervalo do tipo $\Delta$ definimos um ramo da aplicação de Markov $F_{1}$, 
a saber, $F_{1 \mid \Delta}=\phi_{0 \mid \Delta}=f_{\mid \Delta}^{n_{\Delta}}$ o qual é um difeomorfismo sobre $\delta_{1}$ ou $\gamma_{1}$. Neste primeiro passo temos na verdade que $\delta_{1}=\delta_{0}$ e que existem apenas 2 intervalos do tipo $\Delta$ contidos em $\gamma_{0}$ e sua imagem por $F_{1}$ é $\delta_{1}$.

Os intervalos $\delta_{1}$ e $\gamma_{1}$ contêm pontos críticos de $\phi_{0}$ e será necessário subdividí-los na próxima etapa. Para isto definimos $h_{1}=\phi_{0 \mid \delta_{1}}$ e $g_{1}=\phi_{0 \mid \gamma_{1}}$.

Subdividindo $\delta_{1} \cup \gamma_{1}$ : para subdividirmos $\delta_{1} \cup \gamma_{1}$ consideramos o domínio de $F_{1} \circ h_{1}$. Observamos que a imagem de $g_{1}$ não intercepta o domínio de $F_{1}$ (veja figura 3.1) e por isto não consideramos $F_{1} \circ g_{1}$ também. Então apenas $\delta_{1}$ será subdividido nesta etapa. Definimos a partição

$$
\eta_{1}: \delta_{1} \cup \gamma_{1}=(\cup \Delta) \cup \delta_{2} \cup \gamma_{2}
$$

onde $\gamma_{1}=\gamma_{2}$ e $\Delta \subset \delta_{1}$ denota intervalos onde $F_{1} \circ h_{1}$ é monótona ou intervalos que são aplicados difeomorficamente por $h_{1}$ sobre $\gamma_{1}$. Nestes novos intervalos $\Delta \subset \delta_{1}$, $F_{1} \circ h_{1}$ ou $h_{1}$ define uma aplicação de Markov que denotamos por $F_{2}$. Nos intervalos $\Delta$ previamente construídos definimos $F_{2}:=F_{1}$. Observamos que a imagem de um ramo $F_{1 \mid \Delta}$ é $\delta_{1}$ ou $\gamma_{1}$.

Os intervalos $\delta_{1}$ e $\gamma_{1}$ contêm pontos críticos e precisamos subdividí-los na etapa seguinte. Para isto definimos nestes intervalos as aplicações $h_{2}=F_{1} \circ h_{1 \mid \delta_{2}}$ e $g_{2}=g_{1}$. Observamos que $h_{2}$ e $g_{2}$ coincidem com a aplicação de primeiro retorno $\phi_{1}$, restrita aos seus respectivos domínios.

Temos agora uma nova partição de $[0,1]$, a saber:

$$
\xi_{2}:[0,1]=(\cup \Delta) \cup \delta_{2} \cup \gamma_{2} .
$$

Paralelamente estão definidos difeomorfismos de cada intervalo $\Delta$ sobre $\delta_{1}$ ou $\gamma_{1}$. Estes difeomorfismos são ramos de uma aplicação de Markov $F_{2}$. Também estão definidas as aplicações unimodais $h_{2}$ e $g_{2}$ cujos domínios são $\delta_{2}$ e $\gamma_{2}$, respectivamente.

A próxima partição que definiremos será mais um passo na construção por indução de uma seqüência de partições $\xi_{n}$ e de uma seqüência de aplicações de Markov $F_{n}$. A partição $\xi$ que queremos e a aplicação de Markov $F$ serão definidas posteriormente como um limite. Iniciamos pela subdivisão de $\delta_{2} \cup \gamma_{2}$.

Subdividindo $\delta_{2} \cup \gamma_{2}$ : para subdividirmos $\delta_{2} \cup \gamma_{2}$ observamos que $h_{2}(c) \in U_{2}^{c} \subset \delta_{1}$ e $g_{2}(d) \in U_{2}^{d} \subset \delta_{1}$, e que $U_{2}^{c}$ e $U_{2}^{d}$ são pré-imagens de $\gamma_{1}$ por $h_{1}$. Definimos uma partição preliminar

$$
\eta_{2}: \delta_{2} \cup \gamma_{2}=(\cup \Delta) \cup\left(\cup \delta_{2}^{-1}\right) \cup \delta_{3} \cup \gamma_{3}
$$


onde $\Delta$ denota intervalos que são aplicados por $F_{2} \circ h_{2}$ ou $F_{2} \circ g_{2}$ difeomorficamente sobre $\delta_{1}$ ou $\gamma_{1}, \delta_{2}^{-1}$ denota intervalos que são aplicados por $h_{1}$ ou $g_{1}$ difeomorficamente sobre $\delta_{3}, \delta_{3}=h_{2}^{-1}\left(U_{2}^{c}\right)$ e $\gamma_{3}=g_{2}^{-1}\left(U_{2}^{d}\right)$. Devido ao fato de que as imagens de $h_{2}$ e $g_{2}$ não intercectam $\gamma_{2}$ não temos os intervalos do tipo $\gamma_{2}^{-1}$.

Nestes novos intervalos $\Delta, F_{2} \circ h_{2}$ ou $F_{2} \circ g_{2}$ definem uma aplicação de Markov que denotamos por $F_{3}$. Nos intervalos $\Delta$ previamente construídos definimos $F_{3}:=$ $F_{2}$. Os intervalos $\delta_{3}$ e $\gamma_{3}$ contém pontos críticos e deverão ser subdivididos na etapa seguinte. Para isto definimos nestes intervalos $h_{3}=h_{1} \circ h_{2}$ e $g_{3}=h_{1} \circ g_{2}$, respectivamente. Observamos que $h_{3}$ e $g_{3}$ coincidem com a aplicação de primeiro retorno $\phi_{2}$, restritas aos seus respectivos domínios.

Previamente devemos subdividir os intervalos $\delta_{2}^{-1}$.

Subdividindo $\cup \delta_{2}^{-1}$ : observamos que existem 4 intervalos do tipo $\delta_{2}^{-1}$ com 2 deles contidos em $\delta_{2}$ e os outros 2 contidos em $\gamma_{2}$. Estes intervalos são aplicados difeomorficamente sobre $\delta_{2}$ por $h_{2}$. Então o conjunto dos $x \in \delta_{2}^{-1}$ tais que $h_{2}^{n}(x)$ está definida para todo $n \geq 0$ é um conjunto de Cantor, de fato é uma ferradura de Smale. Uma vez que $\delta_{2}^{-1} \cap \delta_{3}=\emptyset$ resulta pelo Teorema 2.5 que este conjunto tem medida de Lebesgue nula. Trata-se na verdade do conjunto dos pontos de $\delta_{2}$ cujas órbitas positivas não interceptam $(\cup \Delta) \cup \delta_{3} \cup \gamma_{3}$. Resulta então que cada intervalo $\delta_{2}$ é uma união (módulo zero) de intervalos do tipo $\Delta$ e $\delta_{3}^{-k}$ sendo que estes primeiros correspondem a pontos que saem de $\delta_{2}$ sem passar por $\delta_{3}$ depois de certo número de iteradas positivas e estes últimos são aplicados por $h_{2}^{k}$ difeomorficamente sobre $\delta_{3}$. Em $\gamma_{2}$ também existem dois intervalos do tipo $\delta_{2}^{-1}$ que são aplicados difeomorficamente sobre $\delta_{2}$ por $g_{2}$. Então temos que

$$
\cup \delta_{2}^{-1}=(\cup \Delta) \cup\left(\cup \delta_{3}^{-k}\right),
$$

onde $\delta_{3}^{-k}$ são intervalos obtidos de $\delta_{3}$ por $k$ iteradas de ramos de $h_{2}^{-1}$ ou por $k-1$ iteradas de ramos de $h_{2}^{-1}$ seguida de uma iterada de um ramo de $g_{2}^{-1}$.

Assim temos uma nova partição de $\delta_{2} \cup \gamma_{2}$ :

$$
\eta_{2}^{\prime}: \delta_{2} \cup \gamma_{2}=(\cup \Delta) \cup\left(\cup \delta_{3}^{-k}\right) \cup \delta_{3} \cup \gamma_{3}
$$

Usando as subdivisões acima podemos descrever uma nova partição que consideraremos como um primeiro passo de indução, a saber:

Indução (passo 1): a partição

$$
\xi_{3}:[0,1]=(\cup \Delta) \cup\left(\cup \delta_{3}^{-k}\right) \cup \delta_{3} \cup \gamma_{3},
$$

é uma partição (módulo zero) de $[0,1]$ tal que: 
- Cada $\Delta$ é um intervalo que é aplicado difeomorficamente sobre $\delta_{1}$ ou $\gamma_{1}$ por $f^{n_{\Delta}}$, para algum $n_{\Delta}$. Definimos então em $\Delta$ um ramo de uma aplicação de Markov $F_{3}$ pondo $F_{3 \mid \Delta}=f_{\mid \Delta}^{n_{\Delta}}$.

- Verifica-se que $h_{3}$ e $g_{3}$ são os dois ramos unimodais da aplicação de primeiro retorno $\phi_{2}$ e estão definidas em $\delta_{3}, \gamma_{3}$, respectivamente.

- Os intervalos $\delta_{3}^{-k}(k \geq 1)$ são dois a dois disjuntos e são aplicados difeomorficamente sobre $\delta_{3}$ pela composição de $k$ funções escolhidas dentre $h_{2}$ e $g_{2}$.

Indução (hipótese de indução): $\operatorname{sejam~} n \geq 4 \mathrm{e}$

$$
\xi_{n-1}:[0,1]=(\cup \Delta) \cup\left(\cup \delta_{n-1}^{-k}\right) \cup\left(\cup \gamma_{n-1}^{-k}\right) \cup \delta_{n-1} \cup \gamma_{n-1}
$$

uma partição (módulo zero) de $[0,1]$ tal que:

- Cada $\Delta$ é um intervalo que é aplicado difeomorficamente sobre $\delta_{1}$ ou $\gamma_{1}$ por $f^{n_{\Delta}}$, para algum $n_{\Delta}$. Definimos então em $\Delta$ um ramo de uma aplicação de Markov $F_{n-1}$ pondo $F_{n-1 \mid \Delta}=f_{\mid \Delta}^{n}$.

- Verifica-se que $h_{n-1}$ e $g_{n-1}$ são ramos unimodais da aplicação de primeiro retorno $\phi_{n-2}$ e estão definidas em $\delta_{n-1}$ e $\gamma_{n-1}$, respectivamente. As suas imagens estão contidas, ambas em $\delta_{n-2}$ ou em $\gamma_{n-2}$.

- Cada $\delta_{n-1}^{-k}$ ou $\gamma_{n-1}^{-k}(k \geq 1)$ é um intervalo que é aplicado difeomorficamente sobre $\delta_{n-1}$ ou $\gamma_{n-1}$, respectivamente, pela composição de $k$ funções escolhidas dentre $h_{i}$ e $g_{i}$, para $i=1, \ldots n-2$.

Para provarmos o passo indutivo, inicialmente consideramos $h_{n-1}$ e $g_{n-1}$ definidas, respectivamente, em $\delta_{n-1}$ e $\gamma_{n-1}$. Decorre do Lema 3.1 que

$$
\gamma_{n-1} \subset h_{n-1}\left(\delta_{n-1}\right), \quad h_{n-1}(c) \in \delta_{n-1}^{-1} \subset \gamma_{n-2} \text { e } g_{n-1}(d) \in \delta_{n-1}^{-1} \subset \gamma_{n-2}
$$

ou

$$
\delta_{n-1} \subset g_{n-1}\left(\gamma_{n-1}\right), \quad h_{n-1}(c) \in \gamma_{n-1}^{-1} \subset \delta_{n-2} \quad \text { e } g_{n-1}(d) \in \gamma_{n-1}^{-1} \subset \delta_{n-2}
$$

Assumimos no que se segue que ocorre este último caso uma vez que o outro é completamente análogo. Portanto podemos definir $\delta_{n} \ni c$ e $\gamma_{n} \ni d$ que são respectivamente componentes conexas de $h_{n-1}^{-1}\left(\gamma_{n-1}^{-1}\right)$ e de $g_{n-1}^{-1}\left(\gamma_{n-1}^{-1}\right)$. Nestes intervalos definimos, respectivamente, $h_{n}:=h_{n-2} \circ h_{n-1}$ e $g_{n}:=h_{n-2} \circ g_{n-1}$ que são extensões da transformação de primeiro retorno $\phi_{n}$. Agora subdividimos $\delta_{n-1} \cup \gamma_{n-1}$ e posteriormente cada intervalo do tipo $\delta_{n-1}^{-k}$ ou $\gamma_{n-1}^{-k}$. 
Subdividindo $\delta_{n-1} \cup \gamma_{n-1}$ : definimos uma partição $\eta_{n-1}$ de $\delta_{n-1} \cup \gamma_{n-1}$ usando as pré-imagens dos intervalos de $\xi_{n-1}$ por $h_{n-1}$ ou por $g_{n-1}$.

$$
\eta_{n-1}: \delta_{n-1} \cup \gamma_{n-1}=(\cup \Delta) \cup\left(\cup \delta_{n-1}^{-k}\right) \cup\left(\cup \gamma_{n-1}^{-k}\right) \cup \delta_{n} \cup \gamma_{n}
$$

Subdividindo $\left(\cup \delta_{n-1}^{-k}\right) \cup\left(\cup \gamma_{n-1}^{-k}\right)$ : para implementarmos esta subdivisão observamos que as órbitas positivas dos pontos de $\left(\cup \delta_{n-1}^{-k}\right) \cup\left(\cup \gamma_{n-1}^{-k}\right)$ por iterações das funções $h_{i}$ e $g_{i}$ (para $\left.i=1, \ldots n-2\right)$ podem interceptar intervalos $\Delta \in \xi_{n-1}$ sem antes passar por $\delta_{n} \cup \gamma_{n}$ ou vice-versa. Decorre então do Teorema 2.5 que $\left(\cup \delta_{n-1}^{-k}\right) \cup\left(\cup \gamma_{n-1}^{-k}\right)$ é uma união (módulo zero) de intervalos do tipo $\Delta, \delta_{n}^{-k}$ ou $\gamma_{n}^{-k}$. Sendo que estes primeiros correspondem a pontos que visitam (sem passar por $\delta_{n} \cup \gamma_{n}$ ) intervalos do tipo $\Delta$ previamente construídos. Estes últimos são aplicados difeomorficamente sobre $\delta_{n}$ ou $\gamma_{n}$ por $k$ iteradas de funções escolhidas dentre $h_{i}$ e $g_{i}$ para $i=0, \ldots n$ e dão origem a intervalos que denotamos por $\delta_{n}^{-k}$ ou $\gamma_{n}^{-k}$. Assim temos uma partição de $\left(\cup \delta_{n-1}^{-k}\right) \cup\left(\cup \gamma_{n-1}^{-k}\right)$, a saber:

$$
\eta_{n-1}^{\prime}:\left(\cup \delta_{n-1}^{-k}\right) \cup\left(\cup \gamma_{n-1}^{-k}\right)=\cup \Delta \cup\left(\cup \delta_{n}^{-j}\right) \cup\left(\cup \gamma_{n}^{-j}\right) \cup \delta_{n} \cup \gamma_{n}
$$

Usando as subdivisões acima definimos uma nova partição (módulo zero) $\xi_{n}$ de $[0,1]$ :

$$
\xi_{n}:[0,1]=(\cup \Delta) \cup\left(\cup \delta_{n}^{-k}\right) \cup\left(\cup \gamma_{n}^{-k}\right) \cup \delta_{n} \cup \gamma_{n} .
$$

Cada intervalo $\Delta$ é aplicado difeomorficamente sobre $\delta_{1}$ ou $\gamma_{1}$ por uma iterada conveniente de $f$ e define nestes intervalos ramos de uma aplicação de Markov $F_{n}$ induzida por $f$. Com isto finalizamos a indução. Então $\xi$ é definida tomando os intervalos $\Delta$ de cada partição $\xi_{n}$, mais precisamente temos

$$
\xi:=\cup_{n \geq 0}\left\{\Delta \mid \Delta \in \xi_{n}\right\} .
$$

Observamos que ainda precisamos provar que $\xi$ é uma partição módulo zero de $[0,1]$.

Paralelamente definimos uma aplicação de Markov $F$ pondo

$$
F(x):=\lim _{n \rightarrow \infty} F_{n}(x),
$$

para cada $x \in \cup_{\Delta \in \xi} \Delta$. Observamos que a seqüencia $F_{n}(x)$ estabiliza-se, ou seja, se $x \in \Delta \in \xi_{n}$ então $F_{n+i}(x)=F_{n}(x)$ para todo $i \geq 0$. Isto garante a existência do limite acima.

Para cada $n \geq 0$ fixo definimos 


$$
\mathcal{S}_{n}:=\left(\cup \delta_{n}^{-k}\right) \cup\left(\cup \gamma_{n}^{-k}\right) \cup \delta_{n} \cup \gamma_{n}
$$

A seqüência $\mathcal{S}_{n}$ é uma seqüência encaixante, ou seja, $\mathcal{S}_{n+1} \subset \mathcal{S}_{n}$. Definimos também

$$
\mathcal{S}:=\cap_{n=0}^{\infty} \mathcal{S}_{n}
$$

O conjunto $\mathcal{S}$ está contido no complementar em $[0,1]$ do domínio da aplicação $F$. Será necessário provarmos, o que faremos na próxima seção, que o mesmo tem medida de Lebesgue nula. No momento enunciamos apenas um lema de natureza topológica decorrente da construção da partição $\xi$.

Lema 4.1. Uma aplicação bimodal $f$ como no Lema 3.1 que não possui pontos periódicos atratores e intervalos errantes induz uma aplicação de Markov F com partição imagem finita (os intervalos $\delta_{1}$ e $\gamma_{1}$ definidos acima) definida em um subconjunto aberto e denso do intervalo $[0,1]$.

Demonstração: Da hipótese de que $f$ não possui pontos periódicos atratores e intervalos errantes (hipótese esta que se verifica no caso de polinômios cúbicos como no Lema 3.1) decorre que o conjunto $\mathcal{S}$ construído acima possui interior vazio. Portanto $\cup_{\Delta \in \xi} \Delta \subset[0,1]$ é denso em $[0,1]$. Os intervalos $\Delta$ são abertos com interior dois a dois disjuntos, por construção. Também por construção a aplicação $F: \cup_{\Delta \in \xi} \Delta \rightarrow \delta_{1} \cup \gamma_{1}$ que em cada intervalo $\Delta$ coincide com uma iterada $f^{n_{\Delta}}$ é uma aplicação de Markov induzida por $f$ cuja partição imagem é $\left\{\delta_{1}, \gamma_{1}\right\}$.

Lema 4.2. Seja I um intervalo, $I \subset[f(d), f(c)]$. Então $\bigcup_{j=1}^{\infty} f^{j}(I)=[f(d), f(c)]$.

Demonstração: Como $f$ nấo possui intervalos errantes, existe $j \geq 0$ para o qual existe $k \geq 0$ tal que $f^{j}(I) \cap f^{j+k}(I) \neq \emptyset$. Seja $J=\bigcup_{i=1}^{\infty} f^{j+i k}(I)$. Observamos que $f^{k}(J) \subset J$ e $f^{k}$ não é periódica pois se fosse, $f$ teria um ponto periódico atrator. Assumimos, então que $c \in J \cup f(J) \cup \ldots \cup f^{k}(J)$. Portanto $\omega(c) \subset$ $\bar{J} \cup f(\bar{J}) \cup \ldots \cup f^{k}(\bar{J})$ e como $d \in \omega(c)$ temos $\omega(d) \subset \bar{J} \cup f(\bar{J}) \cup \ldots \cup f^{k}(\bar{J})$. Logo, $f(c), f(d) \in \omega(c) \subset \bar{J} \cup f(\bar{J}) \cup \ldots \cup f^{k}(\bar{J})$ e, como $f$ é não renormalizável, temos $\bar{J}=[f(d), f(c)]$ e o lema segue.

Proposição 4.3. O intervalo $A=[f(d), f(c)]$ é o atrator topológico de $f$.

Demonstração: Como $f$ não possui atratores periódicos e é não renormalizável, sabemos (veja [MSt]) que os atratatores topológicos de $f$ devem ser intervalos. Como $c$ e $d$ pertencem a um mesmo conjunto minimal, temos que $f$ possui um 
único atrator. Observamos, então que $A$ é positivamente invariante e pelo Lema 4.1 sua bacia de atração é um conjunto de segunda categoria de Baire em $[0,1]$. Além disso, o Lema 4.2 garante que qualquer intervalo propriamente contido em $A$ tem a mesma bacia de atração.

\subsection{Decaimento de Geometria e Hiperbolicidade Induzida}

Visando provar a hiperbolicidade da aplicação de Markov construída na seção anterior introduziremos alguns fatos decorrentes do decaimento exponencial de geometria. Provaremos a hiperbolicidade de $F$ usando técnicas descritas em [JS]. Estaremos assumindo nesta seção que existem constantes $A>0$ e $\lambda>1$ tais que $\delta_{n}$ e $\gamma_{n}$ estão $A \lambda^{n}$-centralizados em $\delta_{n-1}$ e $\gamma_{n-1}$, respectivamente. Isto implica em particular que

$$
\frac{\left|\delta_{n}\right|}{\left|\delta_{n-1}\right|} \leq \frac{1}{1+2 A \lambda^{n}} \quad \text { e } \quad \frac{\left|\gamma_{n}\right|}{\left|\gamma_{n-1}\right|} \leq \frac{1}{1+2 A \lambda^{n}}
$$

Sabemos que cada intervalo do tipo $\delta_{n}^{-k}$ (ou $\gamma_{n}^{-k}$ ) pertencente à partição $\xi_{n}$ é aplicado difeomorficamente sobre $\delta_{n}\left(\right.$ ou $\left.\gamma_{n}\right)$ por $\varphi_{\mid \delta_{n}^{-k}}$ (ou $\varphi_{\mid \gamma_{n}^{-k}}$ ) a qual é uma composição de $k$ funções escolhidas dentre $h_{i}$ e $g_{i}$, para $i=0, \ldots, n-1$.

Lema 4.4. Para todo $\delta_{n}^{-k}, \gamma_{n}^{-k} \in \xi_{n}$ os difeomorfismos $\varphi_{\mid \delta_{n}^{-k}}: \delta_{n}^{-k} \rightarrow \delta_{n}$ e $\varphi_{\mid \gamma_{n}^{-k}}$ : $\gamma_{n}^{-k} \rightarrow \gamma_{n}$ são $A \lambda^{n}$-extensíveis e têm distorção limitada por $\left(1+\frac{1}{A \lambda^{n}}\right)^{2}$.

Demonstração: De fato, basta observarmos que, pela construção da partição $\xi$, cada $\delta_{n}^{-k} \in \xi_{n}$ está contido em uma pré-imagem de $\delta_{n-1}^{-k}$ (não necessariamente em $\xi_{n-1}$ ) e o difeomorfismo $\varphi$ está definido neste intervalo, cuja imagem é $\delta_{n-1}$. A mesma observação é verdadeira para $\gamma_{n}^{-k}$. Como $\frac{\left|\delta_{n}\right|}{\left|\delta_{n-1}\right|}$ e $\frac{\left|\gamma_{n}\right|}{\left|\gamma_{n-1}\right|}$ decrescem exponencialmente, ou melhor, são majorados por $\left(1+A \lambda^{n}\right)^{-1}$, o lema segue.

Comos observamos anteriormente, decorre do Lema 3.1 que, para $n \geq 2$,

$$
\gamma_{n-1} \subset h_{n-1}\left(\delta_{n-1}\right), h_{n-1}(c) \in \delta_{n-1}^{-1} \subset \gamma_{n-2} \text { e } g_{n-1}(d) \in \delta_{n-1}^{-1} \subset \gamma_{n-2}
$$

ou

$$
\delta_{n-1} \subset g_{n-1}\left(\gamma_{n-1}\right), \quad h_{n-1}(c) \in \gamma_{n-1}^{-1} \subset \delta_{n-2} \quad \text { e } g_{n-1}(d) \in \gamma_{n-1}^{-1} \subset \delta_{n-2} .
$$

Definimos então, $W_{1}(c):=\gamma_{0}$ e $W_{1}(d):=h_{0}^{-1}\left(\gamma_{1}\right)$ e, para $n \geq 2, W_{n}(c):=$ $h_{n-1}^{-1}\left(\delta_{n-2}^{-1}\right)$ e $W_{n}(d):=g_{n-1}^{-1}\left(\delta_{n-2}^{-1}\right)$ se ocorrer o primeiro caso. Se ocorrer o 
segundo caso definimos $W_{n}(c):=h_{n-1}^{-1}\left(\gamma_{n-2}^{-1}\right)$ e $W_{n}(d):=g_{n-1}^{-1}\left(\gamma_{n-2}^{-1}\right)$. Estes intervalos $W_{n}(c)$ e $W_{n}(d)$ são vizinhanças de $\delta_{n}$ e $\gamma_{n}$, respectivamente.

Lema 4.5. Para todo $n \geq 1$ as aplicações unimodais $h_{n}$ e $g_{n}$ possuem $A \lambda^{n}$ extensões unimodais $\widehat{h_{n}}$ e $\widehat{\mathrm{g}_{n}}$. Estas extensões estão definidas, respectivamente, nos intervalos $W_{n}(c)$ e $W_{n}(d)$ e são tais que $\widehat{h_{n}}\left(\partial W_{n}(c)\right)$ e $\widehat{g}_{n}\left(\partial W_{n}(d)\right)$ estão em $\partial \delta_{n-2}$ ou em $\partial \gamma_{n-2}$, para $n \geq 2$. Além disso, para cada $n \geq 1$, existem aplicaçôes monótonas $H_{n}$ e $G_{n}$ tais que

$$
\widehat{h_{n}}=H_{n} \circ f \quad e \quad \widehat{g_{n}}=G_{n} \circ f
$$

com

$\operatorname{para} x, y \in \delta_{n} e$

$$
\left(1+\frac{1}{A \lambda^{n}}\right)^{-2} \leq \frac{D H_{n}(f(x))}{D H_{n}(f(y))} \leq\left(1+\frac{1}{A \lambda^{n}}\right)^{2}
$$

$$
\left(1+\frac{1}{A \lambda^{n}}\right)^{-2} \leq \frac{D G_{n}(f(x))}{D G_{n}(f(y))} \leq\left(1+\frac{1}{A \lambda^{n}}\right)^{2}
$$

para $x, y \in \gamma_{n}$.

Demonstração: Vamos usar indução em $n$. Para $n=1$, temos $h_{1}=f \circ f$ e $g_{1}=f \circ f$ e assim o lema segue. Assumindo-o verdadeiro para $n$ passamos a proválo para $n+1$. Existem 2 casos a serem considerados que dependem da imagem de $h_{n}$ e de $g_{n}$ estarem em $\delta_{n-1}$ ou em $\gamma_{n-1}$. Assumimos que ocorre este primeiro caso uma vez que o outro segue de modo análogo. Faremos a prova apenas para $h_{n+1}$. Temos que $h_{n+1}=h_{n-1} \circ h_{n}, h_{n}\left(\partial \delta_{n}\right) \subset \partial \delta_{n-1}$ e $h_{n}\left(\delta_{n+1}\right) \subset h_{n-1}^{-1}\left(\gamma_{n}\right) \subset h_{n-1}^{-1}\left(\gamma_{n-1}\right) \subset \delta_{n-1}$. Pela hipótese de indução existe um difeomorfismo $H_{n}$ tal que $h_{n}=H_{n} \circ f$. Como $h_{n-1 \mid h_{n-1}^{-1}\left(\gamma_{n-1}\right)}$ é monótona, definindo $H_{n+1}:=h_{n-1} \circ H_{n}$ resulta que $h_{n+1}=H_{n+1} \circ f$ em todo o intervalo $W_{n+1}(c)$ definido anteriormente. A imagem de $H_{n+1}$ é pelo menos o intervalo $\gamma_{n-1}$ enquanto que a imagem de $h_{n+1}$ está contida em $\gamma_{n}$. Como $\frac{\left|\delta_{n}\right|}{\left|\delta_{n-1}\right|}$ e $\frac{\left|\gamma_{n}\right|}{\left|\gamma_{n-1}\right|}$ são majorados por $\left(1+A \lambda^{n}\right)^{-1}$ o lema segue.

Corolário 4.6. Existe um número real $C_{1}>0$ tal que

1. Para $n \geq 0$ e $x \in \delta_{n}$ verifica-se que

$$
\left(1+\frac{C_{1}}{\lambda^{n}}\right)^{-3}\left|D^{2} h_{n}(c)\right||x-c| \leq\left|D h_{n}(x)\right| \leq\left(1+\frac{C_{1}}{\lambda^{n}}\right)^{3}\left|D^{2} h_{n}(c)\right||x-c| .
$$

2. Para $n \geq 0$ e $x \in \gamma_{n}$ verifica-se que

$$
\left(1+\frac{C_{1}}{\lambda^{n}}\right)^{-3}\left|D^{2} g_{n}(d)\right||x-d| \leq\left|D g_{n}(x)\right| \leq\left(1+\frac{C_{1}}{\lambda^{n}}\right)^{3}\left|D^{2} g_{n}(d)\right||x-d| .
$$


Demonstração: Provaremos apenas a primeira afirmação, dado que a segunda prova-se de maneira análoga. Pelo Lema 4.5 temos que $h_{n}$ é $A \lambda^{n}$-extendível e $h_{n}=H_{n} \circ f$, onde $H_{n}$ é um difeomorfismo $A \lambda^{n}$-extendível e, portanto com distorção limitada por $\left(1+\frac{1}{A \lambda^{n}}\right)^{2}$. Como

$$
\frac{\left|\delta_{n}\right|}{\left|\delta_{n-1}\right|} \leq \frac{1}{1+2 A \lambda^{n}}
$$

em particular $\left|\delta_{n}\right| \leq\left(2 A \lambda^{n}\right)^{-1}\left|\delta_{0}\right|$. Sendo 2 a ordem do ponto crítico $c$ concluímos que existe constante $C_{0}<\infty$ tal que para todo $x \in \delta_{n}$ verifica-se que

$$
\left(1+\frac{C_{0}}{\lambda^{n}}\right)^{-1}\left|D^{2} f(c)\right||x-c| \leq|D f(x)| \leq\left(1+\frac{C_{0}}{\lambda^{n}}\right)\left|D^{2} f(c) \| x-c\right| .
$$

Como $D^{2} h_{n}(c)=D H_{n}(f(c)) D^{2} f(c)$ e

$$
\frac{\left|D H_{n}(f(x))\right|}{\left|D H_{n}(f(c))\right|} \leq\left(1+\frac{1}{A \lambda^{n}}\right)^{2}
$$

o lema segue.

Corolário 4.7. Existe constante $C_{2}<\infty$ tal que para todo $n \geq 0$ verifica-se que

$$
\frac{\left|\delta_{n}\right|}{\left|W_{n}(c)\right|} \leq \frac{C_{2}}{\left(1+2 A \lambda^{n}\right)^{\frac{1}{2}}}, \quad \frac{\left|W_{n}(c)\right|}{\left|\delta_{n-1}\right|} \leq \frac{C_{2}}{\lambda^{\frac{n}{2}}}, \quad \frac{\left|\gamma_{n}\right|}{\left|W_{n}(d)\right|} \leq \frac{C_{2}}{\left(1+2 A \lambda^{n}\right)^{\frac{1}{2}}} \quad \text { e } \frac{\left|W_{n}(d)\right|}{\left|\gamma_{n-1}\right|} \leq \frac{C_{2}}{\lambda^{\frac{n}{2}}} .
$$

Demonstração: Vamos demonstrar somente a primeira e a segunda desigualdade, pois as demais seguem a partir de argumentos semelhantes. Assumimos, também, que $\delta_{n}=h_{n-1}^{-1}\left(\gamma_{n-1}^{-1}\right)$.

Pelo Corolário 4.6, integrando-se $D h_{n-1}$ em $\delta_{n}$, temos

$$
\frac{\left|\delta_{n}\right|}{\left|W_{n}(c)\right|} \leq\left(1+\frac{C}{\lambda^{n-1}}\right)^{3}\left(\frac{\left|\gamma_{n-1}^{-1}\right|}{\left|\gamma_{n-2}^{-1}\right|}\right)^{1 / 2}
$$

para alguma constante $C>0$. Usando então o lema de Koebe macroscópico (Lema 2.2) para $h_{n-2 \mid \gamma_{n-2}^{-1}}$, obtemos finalmente

$$
\frac{\left|\delta_{n}\right|}{\left|W_{n}(c)\right|} \leq \frac{C_{2}}{\left(1+2 A \lambda^{n}\right)^{1 / 2}},
$$

para uma constante $C_{2}^{\prime}>0$, o que demonstra a primeira desigualdade.

Para a demonstração da segunda desigualdade, devemos considerar duas situações possíveis : 
1. $h_{n-2}\left(\delta_{n-2}\right)$ é uma $A \lambda^{\frac{n-2}{2}}$-vizinhança de $\gamma_{n-2}$. Neste caso, usamos o Lema 2.2 e obtemos que $\delta_{n-2}$ é uma $A \lambda^{\frac{n-2}{2}}$-vizinhança de $h_{n-2}^{-1}\left(\gamma_{n-2}\right)=\gamma_{n-2}^{-1}$. Então, novamente pelo Corolário 4.6 temos

$$
\frac{\left|W_{n}(c)\right|}{\left|\delta_{n-1}\right|} \leq \frac{C_{2}}{\lambda^{\frac{n}{2}}}
$$

para uma constante $C_{2}>0$;

2. $h_{n-2}\left(\delta_{n-2}\right)$ não é uma $A \lambda^{\frac{n-2}{2}}$-vizinhança de $\gamma_{n-2}$, ou seja, há pelo menos uma das componentes conexas de $h_{n-2}\left(\delta_{n-2}\right) \backslash \gamma_{n-2}$ com comprimento menor que $A \lambda^{\frac{n-2}{2}}\left|\gamma_{n-2}\right|$. Isso pode ocorrer somente com a componente que contém $h_{n-2}(c)$. Pelo Corolário 4.6, verifica-se que

$$
\frac{\left|\gamma_{n-2}^{-1}\right|}{\left|\delta_{n-2}\right|} \leq C \frac{\left(\left(1+A \lambda^{\frac{n-2}{2}}\right)\left|\gamma_{n-2}\right|\right)^{\frac{1}{2}}}{\left|h_{n-2}\left(\delta_{n-2}\right)\right|^{\frac{1}{2}}} \leq C \frac{\left(1+A \lambda^{\frac{n-2}{2}}\right)^{\frac{1}{2}}}{\left(1+A \lambda^{n-2}\right)^{\frac{1}{2}}}
$$

para alguma constante $C>0$. Usando novamente o Corolário 4.6, temos finalmente

$$
\frac{\left|W_{n}(c)\right|}{\left|\delta_{n-1}\right|} \leq \frac{C_{2}}{\lambda^{\frac{n}{2}}}
$$

para uma constante $C_{2}>0$.

Avaliaremos em seguida, qual a proporção que os intervalos $\Delta$ que surgem em cada etapa da construção da partição $\xi$ representa em $[0,1]$. Para isso, definimos

$$
\Gamma_{n}:=\cup_{\Delta \in \xi_{n}} \Delta, \quad \Gamma_{n}(c):=W_{n}(c) \cap \Gamma_{n} \quad \text { e } \quad \Gamma_{n}(d):=W_{n}(d) \cap \Gamma_{n} .
$$

Também definimos

$$
D_{n}:=\min \left\{\frac{\left|\Gamma_{n}(c)\right|}{\left|W_{n}(c)\right|}, \frac{\left|\Gamma_{n}(d)\right|}{\left|W_{n}(d)\right|}\right\}
$$

Lema 4.8. Existe um número real $C_{3}>0$ tal que para todo $n \geq 0$ verifica-se que

$$
D_{n+1} \geq \frac{\lambda^{\frac{n}{2}}}{\left(C_{3}+\lambda^{\frac{n}{2}}\right)} D_{n}
$$


Demonstração: Observamos que cada $\delta_{n}^{-k}$ ou $\gamma_{n}^{-k}$ da partição $\xi_{n}$ faz parte de uma tripla

$$
\delta_{n}^{-k} \subset W_{n}^{-k}(c) \subset \delta_{n-1}^{-k} \quad \text { ou } \quad \gamma_{n}^{-k} \subset W_{n}^{-k}(d) \subset \gamma_{n-1}^{-k} .
$$

Aqui $\delta_{n-1}^{-k}$ e $\gamma_{n-1}^{-k}$ podem não ser elementos da partição $\xi_{n-1}$ e estarem contidos em $\delta_{n} \cup \gamma_{n}$ ou em $\delta_{n-1} \cup \gamma_{n-1}$. Cada uma destas triplas é aplicada difeomorficamente sobre a tripla $\delta_{n} \subset W_{n}(c) \subset \delta_{n-1}$ ou sobre $\gamma_{n} \subset W_{n}(d) \subset \gamma_{n-1}$, respectivamente. Decorre do Corolário 4.7 que a distorção destes difeomorfismos em $W_{n}^{-k}(c)$ ou $W_{n}^{-k}(d)$ é limitada por $\left(1+\frac{B}{\lambda^{\frac{n}{2}}}\right)^{2}$, onde $B<\infty$ independe de $n$. Cada intervalo $W_{n}^{-k}(c)$ ou $W_{n}^{-k}(d)$ contém a respectiva pré-imagem de $\Gamma_{n}(c)$ ou $\Gamma_{n}(d)$ que denotamos por $\Gamma_{n}^{-k}(c)$ ou $\Gamma_{n}^{-k}(d)$. Temos então que

$$
\frac{\left|\Gamma_{n}^{-k}(c)\right|}{\left|W_{n}^{-k}(c)\right|} \geq\left(1+\frac{B}{\lambda^{\frac{n}{2}}}\right)^{-2} \frac{\left|\Gamma_{n}(c)\right|}{\left|W_{n}(c)\right|} \quad \text { e } \quad \frac{\left|\Gamma_{n}^{-k}(d)\right|}{\left|W_{n}^{-k}(d)\right|} \geq\left(1+\frac{B}{\lambda^{\frac{n}{2}}}\right)^{-2} \frac{\left|\Gamma_{n}(d)\right|}{\left|W_{n}(d)\right|} .
$$

Isto e o fato das pré-imagens de $\Gamma_{n}(c)$ serem disjuntas, garante que

$$
\frac{\left|\Gamma_{n} \cap \delta_{n-1}\right|}{\left|\delta_{n-1}\right|} \geq\left(1+\frac{B}{\lambda^{\frac{n}{2}}}\right)^{-2} D_{n} \quad \text { e } \quad \frac{\left|\Gamma_{n} \cap \gamma_{n-1}\right|}{\left|\gamma_{n-1}\right|} \geq\left(1+\frac{B}{\lambda^{\frac{n}{2}}}\right)^{-2} D_{n}
$$

Agora relembramos que existem 2 triplas especiais que contêm os valores críticos $h_{n}(c)$ e $g_{n}(d)$. Elas são pré-imagens difeomorfas por $h_{n-1}$ de $\gamma_{n} \subset W_{n}(d) \subset \gamma_{n-1}$ ou por $g_{n-1}$ de $\delta_{n} \subset W_{n}(c) \subset \delta_{n-1}$, dependendo da paridade de $n$. Suponhamos que sejam pré-imagens por $h_{n-1}$ de $\gamma_{n} \subset W_{n}(d) \subset \gamma_{n-1}$ e denotêmo-las por

$$
\gamma_{n}^{-1} \subset W_{n}^{-1}(d) \subset \gamma_{n-1}^{-1} \text {. }
$$

Em cada uma destas duas pré-imagens especiais $\gamma_{n}^{-1} \subset \gamma_{n-1}^{-1}$ verifica-se que

$$
\frac{\left|\Gamma_{n} \cap \gamma_{n-1}^{-1}\right|}{\left|\gamma_{n-1}^{-1}\right|} \geq\left(1+\frac{B}{\lambda^{\frac{n}{2}}}\right)^{-2} D_{n}
$$

por 4.1. As pré-imagens, respectivamente, por $h_{n}$ e $g_{n}$ destes $\gamma_{n}^{-1} \subset \gamma_{n-1}^{-1}$ definem os intervalos $\delta_{n+1} \subset W_{n+1}(c)$ e $\gamma_{n+1} \subset W_{n+1}(d)$. Também ficam definidos $\Gamma_{n+1}(c) \subset W_{n+1}(c)$ e $\Gamma_{n+1}(d) \subset W_{n+1}(d)$. Todas as outras triplas são tais que os respectivos intervalos $\delta_{n-1}^{-k}$ e $\gamma_{n-1}^{-k}$ não contêm valores críticos e possuem préimagens difeomorfas por $h_{n}$ e $g_{n}$.

Concluímos que

$$
\frac{\left|\Gamma_{n+1}(c)\right|}{\left|W_{n+1}(c)\right|}=\frac{\left|\Gamma_{n+1}(c)\right|}{\left|W_{n+1}(c) \backslash \delta_{n+1}\right|} \frac{\left|W_{n+1}(c) \backslash \delta_{n+1}\right|}{\left|W_{n+1}(c)\right|} \geq\left(1+\frac{B}{\lambda^{\frac{n}{2}}}\right)^{-2}\left(1-\frac{\left|\delta_{n+1}\right|}{\left|W_{n+1}(c)\right|}\right) D_{n} .
$$


Pelo Lema 4.7 temos que

$$
\frac{\left|\delta_{n+1}\right|}{\left|W_{n+1}(c)\right|} \leq \frac{C_{2}}{\left(1+2 A \lambda^{n+1}\right)^{\frac{1}{2}}}
$$

Logo

$$
\frac{\left|\Gamma_{n+1}(c)\right|}{\left|W_{n+1}(c)\right|} \geq\left(1+\frac{B}{\lambda^{\frac{n}{2}}}\right)^{-2}\left(1-\frac{C_{2}}{\left(1+2 A \lambda^{n+1}\right)^{\frac{1}{2}}}\right) D_{n} .
$$

Disto segue que existe uma constante $C_{3}<\infty$ satisfazendo o lema.

Corolário 4.9. Existe $\theta>0$ tal que $D_{n}>\theta \forall n \geq 1$.

Demonstração: Pelo Lema 4.8, existe $C_{3}>0$ tal que

$$
D_{n+1} \geq \frac{\lambda^{\frac{n}{2}}}{\left(C_{3}+\lambda^{\frac{n}{2}}\right)} D_{n}
$$

Então $D_{n} \geq \prod_{i=1}^{n-1} \frac{\lambda^{\frac{i}{2}}}{\left(C_{3}+\lambda^{\frac{i}{2}}\right)} D_{1}$. Mas,

$$
\left(\prod_{i=1}^{n-1} \frac{\lambda^{\frac{i}{2}}}{\left(C_{3}+\lambda^{\frac{i}{2}}\right)}\right)^{-1}=\prod_{i=1}^{n-1}\left(\frac{C_{3}}{\lambda^{\frac{i}{2}}}+1\right)
$$

$\mathrm{e}$

$$
\log \left(\prod_{i=1}^{n-1}\left(\frac{C_{3}}{\lambda^{\frac{i}{2}}}+1\right)\right)=\sum_{i=1}^{n-1} \log \left(\frac{C_{3}}{\lambda^{\frac{i}{2}}}+1\right)<\infty
$$

pois para $i$ suficientemente grande, $\log \left(\frac{C_{3}}{\lambda^{\frac{i}{2}}}+1\right)<\frac{C_{3}}{\lambda^{\frac{i}{2}}}$. Logo, o lema segue.

Mostremos agora que a aplicação de Markov $F$ está definida em quase todo ponto. Isto é o principal ingrediente para a hiperbolicidade desta aplicação.

Lema 4.10. Seja $\mathcal{S}=\cap_{n=0}^{\infty} \mathcal{S}_{n}$ o subconjunto de $[0,1]$ onde $F$ não está definida. Então $|\mathcal{S}|=0$.

Demonstração: Inicialmente observamos que decorre do Corolário 4.9 que

$$
\frac{\left|\mathcal{S} \cap W_{n}(c)\right|}{\left|W_{n}(c)\right|} \leq \beta \quad \text { e } \quad \frac{\left|\mathcal{S} \cap W_{n}(d)\right|}{\left|W_{n}(d)\right|} \leq \beta,
$$

para algum $\beta<1$. 
Dado $x \in \mathcal{S}$ temos que existem intervalos arbitrariamente pequenos do tipo $W_{n}^{-k}(c)$ ou $W_{n}^{-k}(d)$ que contêm $x$. Como estes intervalos são aplicados em $W_{n}(c)$ ou $W_{n}(d)$ por um difeomorfismo com distorção limitada verifica-se que $\frac{\left|\Gamma_{n}^{-k}(c)\right|}{\left|W_{n}^{-k}(c)\right|} \geq$ $\theta^{\prime}>0$, e $\frac{\left|\Gamma_{n}^{-k}(d)\right|}{\left|W_{n}^{-k}(d)\right|} \geq \theta^{\prime}>0$ ou seja,

$$
\frac{\left|\mathcal{S} \cap W_{n}(c)\right|}{\left|W_{n}(c)\right|} \leq \beta^{\prime}<1 \quad \text { e } \quad \frac{\left|\mathcal{S} \cap W_{n}(d)\right|}{\left|W_{n}(d)\right|} \leq \beta^{\prime}<1 .
$$

Logo, $|\mathcal{S}|=0$.

Demonstração do Teorema A: Seja $F: \cup_{\Delta \in \xi} \Delta \rightarrow \delta_{1} \cup \gamma_{1}$ a aplicação de Markov construída na Seção 4.1 que devido ao Lema 4.10 está definida em um subconjunto de medida total em $[0,1]$. A partição imagem $F(\mathcal{P})=\{F(\Delta)\}_{\Delta \in \xi}$ é finita, na verdade é $\left\{\delta_{1}, \gamma_{1}\right\}$. Como os pontos críticos $c$ e $d$ estão em um conjunto minimal $\Omega$ cuja distância a $\partial \delta_{1} \cup \partial \gamma_{1}$ é maior do que algum $\alpha^{\prime}>0$ resulta que, para algum $\alpha>0$, os ramos de $F$ ou mesmo os de $F^{N}$ são $\alpha$-estendíveis. Temos então que estes ramos têm distorção limitada independentemente de $N$. Uma vez que não existem intervalos errantes ou pontos periódicos atratores, o comprimento de cada componente conexa do domínio de $F^{N}$ tende para zero quando $N \rightarrow$ $\infty$. Concluímos que existe $N \geq 1$ e $a>1$ tais que $\left|D F^{N}(x)\right|>a$ para todo $x$ pertencente ao domínio de $F^{N}$. Assim segue que $F^{N}$ é uma aplicação de Markov hiperbólica e concluímos a prova do Teorema A. Além disto, pelo Teorema $2.7, F^{N}$ possui uma p.i.a.c. ergódica e única.

Demonstração do Teorema B: Para mostrar que $f$ não possui atrator selvagern, vamos mostrar que seu atrator topológico é também seu atrator métrico.

Seja $F^{N}$ a aplicação de Markov hiperbólica dada pelo Teorema A. Seja $\left\{B_{n}\right\}$ uma base enumerável de abertos de $[f(d), f(c)]$ e, para cada $n>0$, seja

$$
T_{n}:=\left\{x \in[0,1] \mid \operatorname{orb}^{+}(x) \cap B_{n}=\emptyset\right\} .
$$

Vamos mostrar que $\left|\bigcup_{n=1}^{\infty} T_{n}\right|=0$. Suponhamos que $\left|T_{n_{0}}\right|>0$ para algum $n_{0}>0$. Tomamos $x \in T_{n_{0}}$, ponto de densidade de $T_{n_{0}}$, tal que $F^{N}(x)$ esteja definida. Então podemos tomar um intervalo $I \subset I_{1} \cup J_{1} \operatorname{com} F^{N}(x) \in I$, de comprimento suficientemente pequeno, tal que

$$
\frac{\left|F^{-N}(I) \cap T_{n_{0}}\right|}{\left|F^{-N}(I)\right|}>0 .
$$


Como $F^{N}$ tem distorção limitada e $F^{N}\left(T_{n_{0}}\right)=T_{n_{0}}$, temos que

$$
\frac{\left|I \cap T_{n_{0}}\right|}{|I|}>0
$$

ou seja, $T_{n_{0}}$ tem medida total em algum $I$ contido na imagem de $F^{N}$. Pelo Lema 4.2 temos que $\bigcup_{j=1}^{\infty} f^{j}(I)=[f(d), f(c)]$. Logo, existe $x \in T_{n_{0}}$ tal que or $b^{+}(x) \cap B_{n} \neq \emptyset$ e portanto, $\left|\bigcup_{n=1}^{\infty} T_{n}\right|=0$. Assim, existe um conjunto de medida total em $[0,1]$ cujas órbitas são densas em $[f(d), f(c)]$ e, conseqüentemente $f$ não possui atrator selvagem. 


\section{Capítulo 5}

\section{Probabilidade Invariante}

Neste capítulo vamos demonstrar a existência de uma medida de probabilidade invariante absolutamente contínua para $f$. Faremos isso de duas maneiras diferentes. Primeiramente usando o Teorema 2.10 que garante a existência de uma p.i.a.c., desde que seja satisfeita uma condição de somabilidade (veja o Lema 5.5 adiante) sobre as órbitas críticas. Uma outra maneira de proceder é considerar a p.i.a.c. $\mu$ associada uma aplicação de Markov induzida por $f$ e provarmos que a média temporal $\sum_{\Delta \epsilon \xi} n_{\Delta}|\Delta|$ é finita (veja o Lema 5.6 adiante), obtendo a medida para $f$ usando o Lema 2.8. Ambas as abordagens dependem essencialmente do comportamento da sequência de aplicações $h_{n}$ e $g_{n}$ construídas anteriormente. A comparabilidade entre os tamanhos dos domínios $\delta_{n}$ e $\gamma_{n}$ destas aplicações desempenha um papel chave para o estudo de propriedades de expansividade das mesmas. Propriedades estas que são o principal ingrediente na primeira abordagem. Para a estimativa da média temporal necessária para a segunda abordagem é necessário um estudo mais profundo da relação entre os tamanhos $|\Delta|$ e os tempos $n_{\Delta}$ para cada componente $\Delta$ do domínio de $F$. Iniciaremos fornecendo as propriedades de expansividade de $h_{n}$ e $g_{n}$ que utilizaremos posteriormente.

\subsection{Expansividade dos Ramos Críticos}

Tanto para demonstrar que $f$ satisfaz as hipóteses do Teorema 2.10 como para mostrar que a média temporal é finita serão necessárias estimativas mais precisas sobre os módulos de $h_{n}$ e $g_{n}$ e sobre a relação entre $\left|\delta_{n}\right|$ e $\left|\gamma_{n}\right|$ para cada $n>0$. O nosso ponto de partida é o Corolário 4.6 mas precisamos de mais algumas informações.

Lema 5.1. Existe um número real $K>0$ tal que 
1. Para todo $n \geq 1$ verifica-se que

$$
\frac{1}{K} \leq \frac{\left|D^{2} h_{n}(c)\right|}{\left|D^{2} g_{n}(d)\right|} \leq K \quad \text { e } \frac{1}{K} \leq \frac{\left|\delta_{n}\right|}{\left|\gamma_{n}\right|} \leq K
$$

2. Para todo $n \geq 1, v \in \delta_{n} \backslash \delta_{n+1}$ e $w \in \gamma_{n} \backslash \gamma_{n+1}$ verificam-se que

$$
\left|D h_{n}(v)\right| \geq K^{-1} \quad \text { e } \quad\left|D g_{n}(w)\right| \geq K^{-1} .
$$

3. Para todo $n \geq 1, v \in \delta_{n} \backslash W_{n+1}(c)$ e $w \in \gamma_{n} \backslash W_{n+1}(d)$ verificam-se que

$$
\left|D h_{n}(v)\right| \geq \frac{1}{K}\left(\frac{\left|\delta_{n-1}\right|}{\mid \delta_{n}} \mid\right)^{\frac{1}{2}} \quad \text { e } \quad\left|D g_{n}(w)\right| \geq \frac{1}{K}\left(\frac{\left|\delta_{n-1}\right|}{\left|\delta_{n}\right|}\right)^{\frac{1}{2}}
$$

Demonstração: Observamos que por um argumento análogo ao usado na prova do Lema 4.5 podemos escrever $h_{n}=\mathcal{H}_{n} \circ h_{1}$ e $g_{n}=\mathcal{G}_{n} \circ g_{1}$ onde $\mathcal{H}_{n}$ e $\mathcal{G}_{n}$ são iteradas apropriados de $f$ que aplicam parte de seus domínios (as quais denotamos por $U_{n}$ e $V_{n}$ respectivamente) difeomorficamente sobre $\delta_{n-1}$ ou $\gamma_{n-1}$. A distorção destes difeomorfismos é limitada por $K_{n}=\left(1+\frac{1}{A \lambda^{n}}\right)^{2}$. Temos ainda, $U_{n}$ e $V_{n}$ são simétricos com respeito ao ponto crítico $c$ no sentido que $f\left(U_{n}\right)=f\left(V_{n}\right)$. Temos então que $D^{2} h_{n}(c)=D \mathcal{H}_{n}\left(h_{1}(c)\right) D^{2} h_{1}(c)$ e supondo que as imagens são $\delta_{n-1}$ (o caso $\gamma_{n-1}$ é análogo) resulta que

$$
\frac{1}{K_{n}} \frac{\left|\delta_{n-1}\right|}{\left|U_{n}\right|}\left|D^{2} h_{1}(c)\right| \leq\left|D^{2} h_{n}(c)\right| \leq K_{n} \frac{\left|\delta_{n-1}\right|}{\left|U_{n}\right|}\left|D^{2} h_{1}(c)\right| .
$$

Analogamente

$$
\frac{1}{K_{n}} \frac{\left|\delta_{n-1}\right|}{\left|V_{n}\right|}\left|D^{2} g_{1}(d)\right| \leq\left|D^{2} g_{n}(d)\right| \leq K_{n} \frac{\left|\delta_{n-1}\right|}{\left|V_{n}\right|}\left|D^{2} g_{1}(d)\right|
$$

e portanto

$$
\frac{1}{K_{n}^{2}} \frac{\left|V_{n}\right|}{\left|U_{n}\right|} \frac{\left|D^{2} h_{1}(c)\right|}{\left|D^{2} g_{1}(d)\right|} \leq \frac{\left|D^{2} h_{n}(c)\right|}{\left|D^{2} g_{n}(d)\right|} \leq K_{n}^{2} \frac{\left|V_{n}\right|}{\left|U_{n}\right|} \frac{\left|D^{2} h_{1}(c)\right|}{\left|D^{2} g_{1}(d)\right|} .
$$

A segunda afirmação da parte 1 segue a partir do fato que $\left|D^{2} h_{n}(c)\right|$ e $\left|D^{2} g_{n}(d)\right|$ são comparáveis e de que $\left|h_{n}\left(\delta_{n}\right)\right|$ e $\left|g_{n}\left(\gamma_{n}\right)\right|$ são também ambos comparáveis a $\left|\delta_{n-1}\right|$ ou a $\left|\gamma_{n-1}\right|$. Para provarmos este último fato relembramos que $h_{n+1}=$ $h_{n-1} \circ h_{n}$ e $g_{n+1}=h_{n-1} \circ g_{n}$ (о caso $h_{n+1}=g_{n-1} \circ h_{n}$ e $g_{n+1}=g_{n-1} \circ g_{n}$ é análogo) e $h_{n}(c), g_{n}(d) \in h_{n-1}^{-1}\left(\gamma_{n}\right)$. Aqui a pré-imagem $h_{n-1}^{-1}\left(\gamma_{n}\right)$ é constituída de duas componentes conexas que denotamos por $[x, y]=\gamma_{n}^{-1}$. Se $\left[z, z^{*}\right]=\delta_{n-1} \mathrm{e}$ $B_{n}=\left(1+\frac{C_{1}}{\lambda^{n}}\right)^{-3}$, do Corolário 4.6 concluímos que 
e também que

$$
|z-x| \geq \frac{\left|h_{n-1}(z)-h_{n-1}(x)\right|}{B_{n-1}|z-c|\left|D^{2} h_{n-1}(c)\right|}
$$

$$
\frac{4\left|h_{n-1}(z)-h_{n-1}(c)\right|}{B_{n-1}|z-c|^{2}} \leq\left|D^{2} h_{n-1}(c)\right| \leq 4 B_{n-1} \frac{\left|h_{n-1}(z)-h_{n-1}(c)\right|}{|z-c|^{2}} .
$$

E isto implica que a distância de $[x, y]=\gamma_{n}^{-1}$ ao bordo de $\delta_{n-1}$ é dada por $|z-x|$ que satisfaz $M_{1} B_{n}^{2}|z-x| \geq\left|\delta_{n-1}\right|$, para alguma constante $M_{1}<\infty$ e independente de $n$. Isto mais o fato de que $h_{n}(c), g_{n}(d) \in h_{n-1}^{-1}\left(\gamma_{n}\right)$ implica que $\left|h_{n}\left(\delta_{n}\right)\right|$ e $\left|g_{n}\left(\gamma_{n}\right)\right|$ são ambos comparáveis a $\left|\delta_{n-1}\right|$. Desta forma a segunda parte segue-se. Passemos agora à estimativa da derivada de $h_{n}$ (a derivada de $g_{n}$ pode ser estimada do mesmo modo). Para $v \in \delta_{n} \backslash \delta_{n+1}$ temos que

$$
\left|D h_{n}(v)\right| \geq B_{n-1}^{-2}\left|D^{2} h_{n}(c)\right|^{\frac{1}{2}}\left|h_{n}(v)-h_{n}(c)\right|^{\frac{1}{2}} .
$$

Sabemos que

$$
\left|D^{2} h_{n}(c)\right| \geq \frac{2\left|\delta_{n-1}\right|}{B_{n}\left|\delta_{n}\right|^{2}} \quad \text { e } \quad\left|h_{n}(v)-h_{n}(c)\right| \geq\left|h_{n}\left(\delta_{n+1}\right)\right| \geq \frac{\left|\gamma_{n}^{-1}\right|}{M_{2}},
$$

para alguma constante $M_{2}<\infty$ e independente de $n$.

Necessitaremos então de uma estimativa para o tamanho do intervalo $[x, y]=$ $\gamma_{n}^{-1}=h_{n-1}^{-1}\left(\gamma_{n}\right)$. Verifica-se que

$$
|x-y| \geq \frac{\left|h_{n-1}(x)-h_{n-1}(y)\right|}{B_{n-1}\left|D^{2} h_{n-1}(c)\right||x-c|} \geq \frac{\left|\gamma_{n}\right|\left|\delta_{n-1}\right|^{2}}{4 B_{n-1}^{2}\left|\gamma_{n-2}\right||x-c|} .
$$

Mas $\left|\delta_{n-1}\right| \geq|x-c|$ e reunindo estas informações obtemos que

$$
\left|D h_{n}(v)\right| \geq\left(\frac{1}{2 M_{2} B_{n} B_{n-1}^{4}}\right)^{\frac{1}{2}} \frac{\left|\delta_{n-1}\right|}{\left|\delta_{n}\right|}\left(\frac{\left|\gamma_{n}\right|}{\left|\gamma_{n-2}\right|}\right)^{\frac{1}{2}} .
$$

A primeira parte deste lema garante que $\left|\delta_{n}\right|$ e $\left|\gamma_{n}\right|$ são comparáveis e usando o decaimento de geometria concluimos a segunda parte. Para provarmos a terceira parte usamos que para $v \in \delta_{n} \backslash W_{n+1}(c)$ vale que

$$
\left|h_{n}(v)-h_{n}(c)\right| \geq\left|h_{n}\left(W_{n+1}(c)\right)\right| \geq \frac{\left|h_{n-1}^{-1}\left(\gamma_{n-1}\right)\right|}{M_{3}},
$$

para alguma constante $M_{3}<\infty$ e independente de $n$. Se $[x, y]=h_{n-1}^{-1}\left(\gamma_{n-1}\right)$ verifica-se que

$$
|x-y| \geq \frac{\left|h_{n-1}(x)-h_{n-1}(y)\right|}{B_{n-1}\left|D^{2} h_{n-1}(c)\right||x-c|} \geq \frac{\left|\gamma_{n-1}\right|\left|\delta_{n-1}\right|^{2}}{4 B_{n-1}^{2}\left|\gamma_{n-2}\right||x-c|} .
$$


Novamente $\left|\delta_{n-1}\right| \geq|x-c|$ e portanto

$$
\left|D h_{n}(v)\right| \geq \frac{\left|\delta_{n-1}\right|}{2 M_{3} B_{n} B_{n-1}^{4}\left|\delta_{n}\right|}\left(\frac{\left|\gamma_{n-1}\right|}{\left|\gamma_{n-2}\right|}\right)^{\frac{1}{2}} \geq \frac{1}{K}\left(\frac{\left|\delta_{n-1}\right|}{\left|\delta_{n}\right|}\right)^{\frac{1}{2}},
$$

para alguma constante $K<\infty$ e independente de $n$ e disto a terceira parte do lema segue-se.

De acordo com o Lema 5.1 as aplicações $h_{n}$ e $g_{n}$ não são necessariamente expansivas nas regiões $W_{n+1}(c) \backslash \delta_{n+1}$ e $W_{n+1}(d) \backslash \gamma_{n+1}$, respectivamente. Isto dificulta a prova do Lema 5.6. No entanto 2 iterações na região $W_{n+1}(c) \backslash \delta_{n+1}$ ou $y \in W_{n+1}(d) \backslash \gamma_{n+1}$ produzirá uma expansão da ordem de pelo menos $\left(\frac{\left|\delta_{n-2}\right|}{\left|\delta_{n-1}\right|}\right)^{\frac{1}{2}}$ Este é o conteúdo do próximo lema.

Lema 5.2. Existe constante $K<\infty$ (a mesma do Lema 5.1) tal que para $v \in$ $W_{n+1}(c) \backslash \delta_{n+1}$ ou $w \in W_{n+1}(d) \backslash \gamma_{n+1}$ verifica-se que

1. Se $h_{n}(v), g_{n}(w) \in \delta_{n-1}$ ocorre que

$$
\left|D\left(h_{n-1} \circ h_{n}\right)(v)\right| \geq \frac{1}{K^{2}}\left(\frac{\left|\delta_{n-2}\right|}{\left|\delta_{n-1}\right|}\right)^{\frac{1}{2}} \quad \text { e }\left|D\left(h_{n-1} \circ g_{n}\right)(w)\right| \geq \frac{1}{K^{2}}\left(\frac{\left|\delta_{n-2}\right|}{\left|\delta_{n-1}\right|}\right)^{\frac{1}{2}} \text {. }
$$

2. Se $h_{n}(v), g_{n}(w) \in \gamma_{n-1}$ ocorre que

$$
\left|D\left(g_{n-1} \circ h_{n}\right)(v)\right| \geq \frac{1}{K^{2}}\left(\frac{\left|\delta_{n-2}\right|}{\left|\delta_{n-1}\right|}\right)^{\frac{1}{2}} \quad \text { e } \quad\left|D\left(g_{n-1} \circ g_{n}\right)(v)\right| \geq \frac{1}{K^{2}}\left(\frac{\left|\delta_{n-2}\right|}{\left|\delta_{n-1}\right|}\right)^{\frac{1}{2}} \text {. }
$$

Demonstração: Faremos apenas a estimativa de $\left|D\left(h_{n-1} \circ h_{n}\right)(v)\right|$ no caso $h_{n}(v) \in$ $\delta_{n-1}$ uma vez que as outras seguem exatamente os mesmos argumentos. Esta estimativa é uma consequência imediata do Lema 5.1, da definição de $W_{n+1}(c)$ que implica que $h_{n}\left(W_{n+1}(c)\right) \subset h_{n-1}^{-1}\left(\gamma_{n-1}\right)$ e do fato que $W_{n}(c) \cap h_{n-1}^{-1}\left(\gamma_{n-1}\right)=\emptyset$.

\subsection{Somabilidade nas Órbitas Críticas}

Nesta seção, mostraremos que $f$ satisfas as condições do Teorema 2.10. Seguiremos aqui os passos usados em [MN] para funções da família quadrática. O lema abaixo tem um papel fundamental.

Lema 5.3. Existe $C>0$ tal que para $n \geq 1$ se $x \in \delta_{n+1} \cup \gamma_{n+1}$ verifica-se que

$$
\left|D f^{s_{n}}(f(x))\right| \geq C \frac{\left|\delta_{n-1}\right|}{\left|\delta_{n}\right|} .
$$


Demonstração: Demonstraremos apenas a afirmação para $x \in \delta_{n+1}$, pois a demonstração para $x \in \gamma_{n+1}$ segue os mesmos passos. Pelo Lema 4.5, para cada $x \in \delta_{n}, h_{n}(x)=H_{n}(f(x))$ e existe $K>0$, independente de $n$, tal que

$$
\frac{1}{K} \leq \frac{\left|D H_{n}(f(x))\right|}{\left|D H_{n}(f(y))\right|} \leq K
$$

para todo $x, y \in \delta_{n}$.

Além disso como $\left|\delta_{n-1}\right|$ e $\left|\gamma_{n-1}\right|$ são comparáveis, Lema 5.1, existe constante $C_{1}>0$ tal que

$$
\left|H_{n}\left(f\left(\delta_{n}\right)\right)\right| \geq C_{1}\left|\delta_{n-1}\right| .
$$

Observamos ainda, que como $c$ tem ordem 2 , existe $C_{3}>0$ tal que

$$
\left|f\left(\delta_{n}\right)\right| \geq C_{3}\left|\delta_{n}\right|^{2}
$$

Pelo mesmo motivo, uma vez que $f\left(H_{n}\left(f\left(\delta_{n+1}\right)\right)\right) \cap\left(\delta_{n} \cup \gamma_{n}\right)=\emptyset$ temos que existe contante $C_{2}$ tal que

$$
\left|D f\left(H_{n}(f(x))\right)\right| \geq C_{2}\left|\delta_{n}\right|
$$

Logo, existe uma constante $C^{\prime}>0$ tal que

$$
\left|D f^{s_{n}}(f(x))\right|=\left|D f\left(H_{n}(f(x))\right)\right|\left|D H_{n}(f(x))\right| \geq C \frac{\left|\delta_{n-1}\right|}{\left|\delta_{n}\right|^{2}}\left|\delta_{n}\right| \geq C \frac{\left|\delta_{n-1}\right|}{\left|\delta_{n}\right|} .
$$

Assim concluímos a demonstração do lema.

Dado $k>0$, vamos estimar $D f^{k}(f(c))$. Observamos que a estimativa de $D f^{k}(f(d))$ pode ser feita da mesma maneira. Fixemos $n_{0}$ para que tenhamos

$$
b:=\sum_{n=n_{0}}^{\infty}\left(\frac{\left|\delta_{n}\right|}{\left|\delta_{n-1}\right|}\right)^{\frac{1}{2}}<1 .
$$

Seja $m>0$ o maior possível tal que $\delta_{n_{0}+m} \cup \gamma_{n_{0}+m}$ é visitado pela órbita de $f(c)$ até o tempo $k-1$.

Definimos

$$
k_{m}:=\max \left\{1 \leq j \leq k \mid f^{j}(c) \in \delta_{n_{0}+m} \cup \gamma_{n_{0}+m}\right\}
$$


Para $i \leq m$ definimos os inteiros $k_{i}$ como a iterada mais alta de $f(c)$, menor ou igual a $k-1$, a interceptar $\delta_{n_{0}+i-1} \cup \gamma_{n_{0}+i-1}$. Assim, se $\left\{k_{i}<j \leq k \mid f^{j}(c) \in\right.$ $\left.\delta_{n_{0}+i} \cup \gamma_{n_{0}+i}\right\}=\emptyset$, então $k_{i-1}=k_{i}$. Caso contrário,

$$
k_{i-1}=\max \left\{k_{i}<j \leq k \mid f^{j}(c) \in \delta_{n_{0}+i-1} \cup \gamma_{n_{0}+i-1}\right\} .
$$

Observação 5.4. Observamos que $k_{m} \leq k_{m-1} \leq \ldots \leq k_{0}$ definidos acima satisfazem $k_{m}=s_{n_{0}+m}$ e $k_{i}-k_{i+1}=0$ ou $k_{i}-k_{i+1}=s_{n_{0}+i}$, pois nunca há dois retornos de $f(c) a \delta_{n_{0}+i} \cup \gamma_{n_{0}+i}$ sem uma passagem por $\delta_{n_{0}+i+1} \cup \gamma_{n_{0}+i+1}$ entre eles. De fato, por $f$ ser um polinômio de Fibonacci, se $f^{k_{i}}(c) \in\left(\delta_{n_{0}+i} \cup \gamma_{n_{0}+i}\right) \backslash\left(\delta_{n_{0}+i+1} \cup \gamma_{n_{0}+i+1}\right)$, então $k_{i-1}=k_{i}$, ou seja, a situação $k_{m}<k_{m-1}<\ldots<k_{0}$ nunca acontece. Ainda, se $k_{i} \neq k_{i+1}$, então $f^{k_{i+1}}(c) \in \delta_{n_{0}+i+1} \cup \gamma_{n_{0}+i+1}$.

Lema 5.5. Seja $f$ um polinômio de Fibonacci bimodal, conforme descrito no capitulo 2 e sejam $c$ e d seus pontos críticos. Existe $L>0$ tal que

$$
\sum_{i=1}^{\infty}\left(\left|D f^{i}(f(c))\right|\right)^{-1 / 2}+\sum_{i=1}^{\infty}\left(\left|D f^{i}(f(d))\right|\right)^{-1 / 2} \leq L .
$$

Demonstração: Com a Observação 5.4 e o Teorema 2.5, podemos estimar $\left|D f^{k}(f(c))\right|$ e obtemos

$$
\left|D f^{k}(f(c))\right|=\left|D f^{k-k_{0}}\left(f^{k_{0}}(f(c))\right)\right|\left|D f^{s_{n_{0}+m}}(f(c))\right| \prod_{\substack{0 \leq i \leq m-1 \\ k_{i} \neq k_{i+1}}}\left|D f^{k_{i}-k_{i+1}}\left(f^{k_{i+1}}(f(c))\right)\right| .
$$

Assim,

$$
\left(\left|D f^{k}(f(c))\right|\right)^{-1 / 2} \leq\left(\left|D f^{k-k_{0}}\left(f^{k_{0}}(f(c))\right)\right|\right)^{-1 / 2} \prod_{\substack{0 \leq i \leq m \\ k_{i} \neq k_{i+1}}}\left(\left|D f^{s_{n_{0}+i}}\left(f^{k_{i+1}}(f(c))\right)\right|\right)^{-1 / 2} .
$$

Como $k-k_{0} \leq s_{n_{0}-1}$, usamos o Lema 5.3 e observamos que fixado $k_{0}$ não há dois produtos à direita da desigualdade com os mesmos fatores. Assim, a soma de todos os diferentes possíveis produtos é majorada por $1+b+b^{2}+\ldots$ Então podemos estimar

$$
\begin{aligned}
\sum_{k=1}^{\infty}\left(\left|D f^{k}(f(c))\right|\right)^{-1 / 2} & \leq C^{-1 / 2} \sum_{i=1}^{s_{n_{0}-1}} \beta^{-i / 2}\left(1+b+b^{2}+\ldots\right) \\
& \leq C^{-1 / 2}\left(\frac{\beta^{-\left(s_{n_{0}}-1\right)}-1}{\beta-1}\right)\left(\frac{1}{1-b}\right)<\infty
\end{aligned}
$$


Observamos que uma estimativa semelhante para

$$
\sum_{k=1}^{\infty}\left(\left|D f^{k}(f(d))\right|\right)^{-1 / 2}
$$

é obtida da mesma maneira.

O Lema 5.5 nos garante que $f$ satisfaz as condições do Teorema 2.10 e portanto possui uma p.i.a.c..

\subsection{A Média Temporal}

Nossa estratégia aqui é a de obter uma p.i.a.c. para $f$ a partir da p.i.a.c. existente para uma aplicação de Markov hiperbólica induzida por $f$. Será necessário provarmos a finitude da média temporal do Lema 2.8. Para isto é suficiente provarmos o Lema 5.6 abaixo sobre a finitude da média temporal em relação à medida de Lebesgue.

Lema 5.6. Seja $F: \cup_{\Delta \in \xi} \Delta \rightarrow I \cup J$ a aplicação de Markov induzida por $f$, com $F_{\mid \Delta}=f_{\mid \Delta}^{n_{\Delta}}$. Existe $L<\infty$ tal que

$$
\sum_{\Delta \in \xi} n_{\Delta}|\Delta| \leq L
$$

Com o Lema 5.2 temos a expansão suficiente para provarmos o Lema 5.6 e obter a probabilidade invariante para $f$. Mas de fato esta expansão só ocorre para $n$ suficientemente grande e isto nos leva a substituir a aplicação de Markov $F$ construída no Capítulo 4 por outra mais conveniente que denotaremos por $\mathcal{F}$. Escolhemos $n_{0}$ suficientemente grande, a ser especificado mais tarde. Então consideramos a partição

$$
\xi_{n_{0}}: \delta_{0} \cup \gamma_{0}=(\cup \Delta) \cup\left(\cup \delta_{n_{0}}^{-k}\right) \cup\left(\cup \gamma_{n_{0}}^{-k}\right) \cup \delta_{n_{0}} \cup \gamma_{n_{0}}
$$

e definimos uma aplicação $\mathcal{F}_{n_{0}}$ do seguinte modo: nos intervalos $\Delta \in \xi_{n_{0}}$ definímola de modo a coincidir com $F_{n_{0}}$ e nos intervalos $\delta_{n_{0}}^{-k}$ e $\gamma_{n_{0}}^{-k}$ definímo-la de modo a coincidir com a aplicação que leva estes intervalos difeomorficamente em $\delta_{n_{0}}$ ou em $\gamma_{n_{0}}$. Continuaremos denotando por $\Delta$ as componentes conexas do domínio de $\mathcal{F}_{n_{0}}$. Assim temos uma partição inicial

$$
\mathcal{P}_{n_{0}}:[0,1]=(\cup \Delta) \cup \delta_{n_{0}} \cup \gamma_{n_{0}}
$$


Utilisando $h_{n}$ e $g_{n}$ para $n \geq n_{0}$ e a mesma construção das sequências $\xi_{n}$ e $F_{n}$ definimos sequências $\mathcal{P}_{n}$ de partições (módulo zero) do intervalo $[0,1]$ e $\mathcal{F}_{n}$. Resulta desta construção, por uma espécie de limite, como no caso da definição de $\xi$ e $F$, uma partição (módulo zero) $\mathcal{P}$ de $[0,1]$ e uma aplicação de Markov $\mathcal{F}$. Esta aplicação de Markov $\mathcal{F}$ assim obtida possui partição imagem finita. A partição imagem resultante é na verdade constituída pelos intervalos $\delta_{1}$ e $\gamma_{1}$ como antes mas surgem os intervalos $\delta_{n_{0}}$ e $\gamma_{n_{0}}$. Da mesma maneira que mostramos que existe $N>0$ para o qual $F^{N}$ é uma aplicação de Markov hiperbólica e possui uma p.i.a.c., podemos mostrar que $\mathcal{F}$ satisfaz estas condições. De fato, como o conjunto limite $\Omega=\omega(c)=\omega(d)$ é minimal teremos que a sua distância ao bordo destes intervalos é positiva. Disto, e usando o mesmo argumento da prova do Teorema A, segue que existe $N$ tal que $\mathcal{F}^{N}$ é hiperbólica e possui uma p.i.a.c. Então a própria $\mathcal{F}$ possui uma p.i.a.c. Sendo assim provaremos o Lema 5.6 para $\mathcal{F}$ o que garantirá que $f$ possui também uma p.i.a.c.

Demonstração do Lema 5.6 para $\mathcal{F}$. Seja $\Delta \in \mathcal{P}_{n_{0}}$ e $n_{\Delta}$ tal que $\mathcal{F}_{\mid \Delta}=f_{\mid \Delta}^{n_{\Delta}}$. Então, pelo Lema 2.6, temos que

$$
\sum_{\Delta \in \mathcal{P}_{n_{0}, n_{\Delta}}=k}|\Delta|
$$

decresce exponencialmente com $k$. Isto implica que

$$
\sum_{\Delta \in \mathcal{P}_{n_{0}}} n_{\Delta}|\Delta|=\sum_{k=1}^{\infty} k \sum_{\Delta \in \mathcal{P}_{n_{0}}, n_{\Delta}=k}|\Delta|
$$

é limitada.

Passamos agora a estimar $\sum_{\Delta \in \mathcal{P}_{n_{0}+1}} n_{\Delta}|\Delta|$. Para isto observamos que $\mathcal{P}_{n_{0}+1}$ é a coleção de todos os intervalos que são aplicados difeomorficamente por $h_{n_{0}}$ ou $g_{n_{0}}$ em algum $\Delta \in \mathcal{P}_{n_{0}}$. Continuamos denotando estes novos intervalos por $\Delta \mathrm{e}$ observamos que cada $\Delta \in \mathcal{P}_{n_{0}}$ tem, no máximo 4 componentes conexas que são intervalos $\Delta \in \mathcal{P}_{n_{0}+1}$.

Dos Lemas 5.1 e 5.2 resulta que

$$
\sum_{\Delta^{\prime} \in \mathcal{P}_{n_{0}+1}^{0}} n_{\Delta^{\prime}}\left|\Delta^{\prime}\right| \leq 4 K^{2}\left(\frac{\left|\delta_{n_{0}-1}\right|}{\left|\delta_{n_{0}-2}\right|}\right)^{\frac{1}{2}} \sum_{\Delta \in \mathcal{P}_{n_{0}}}\left(n_{\Delta}+s_{n_{0}}\right)|\Delta| .
$$

Ainda temos que $n_{\Delta} \geq s_{n_{0}-1}$ para cada $\Delta \in\left(h_{n_{0}}\left(\delta_{n_{0}}\right) \cup g_{n_{0}}\left(\gamma_{0}\right)\right)$ e, portanto, 


$$
\sum_{\Delta^{\prime} \in \mathcal{P}_{n_{0}+1}^{0}} n_{\Delta^{\prime}}\left|\Delta^{\prime}\right| \leq 12 K^{2}\left(\frac{\left|\delta_{n_{0}-1}\right|}{\left|\delta_{n_{0}-2}\right|}\right)^{\frac{1}{2}} \sum_{\Delta \in \mathcal{P}_{n_{0}}} n_{\Delta}|\Delta| .
$$

A nossa estratégia é proceder de modo análogo para obter uma estimativa para $\sum_{\Delta \in \mathcal{P}_{n}} n_{\Delta}|\Delta|$ em função de $\sum_{\Delta \in \mathcal{P}_{n-1}} n_{\Delta}|\Delta|$. Mas para $n>n_{0}+1$ definiremos várias etapas na construção de $\mathcal{P}_{n}$, as quais utilizaremos nas estimativas. Estas etapas estão ilustradas na Figura 5.1.

Definimos $\mathcal{P}_{n}^{0}$ que é a coleção de todos os intervalos que são aplicados difeomorficamente por $h_{n}$ ou $g_{n}$ em algum $\Delta \in \mathcal{P}_{n-1}$. Continuamos denotando estes novos intervalos por $\Delta$. Dos Lemas 5.1 e 5.2 resulta que

$$
\sum_{\Delta \in \mathcal{P}_{n}^{0}} n_{\Delta}|\Delta| \leq 4 K^{2}\left(\frac{\left|\delta_{n-1}\right|}{\left|\delta_{n-2}\right|}\right)^{\frac{1}{2}} \sum_{\Delta \in \mathcal{P}_{n-1}}\left(n_{\Delta}+s_{n}\right)|\Delta| .
$$

Ainda temos que $n_{\Delta} \geq s_{n-1}$, para cada $\Delta \in \mathcal{P}_{n-1}, \Delta \subset\left(h_{n}\left(\delta_{n}\right) \cup g_{n}\left(\gamma_{n}\right)\right)$ e portanto

$$
\sum_{\Delta \in \mathcal{P}_{n}^{0}} n_{\Delta}|\Delta| \leq 12 K^{2}\left(\frac{\left|\delta_{n-1}\right|}{\left|\delta_{n-2}\right|}\right)^{\frac{1}{2}} \sum_{\Delta \in \mathcal{P}_{n-1}} n_{\Delta}|\Delta| .
$$

Indutivamente, para $j \geq 1$, definimos $\mathcal{P}_{n}^{j}$ que é a coleção de todos os intervalos que são aplicados difeomorficamente por $h_{i}$ ou $g_{i}\left(i=n_{0}, \ldots, n\right)$ em algum $\Delta \epsilon$ $\mathcal{P}_{n}^{j-1}$. Cada um destes $\Delta \in \mathcal{P}_{n}^{j-1}$ possui no máximo $4\left(n-n_{0}\right)$ pré-imagens (2 para cada $h_{i}$ ou $\left.g_{i}\right)$ as quais são intervalos $\Delta^{\prime} \in \mathcal{P}_{n}^{j}$. Dos Lemas 5.1 e 5.2 resulta que cada pré-imagem por um ramo de $h_{i}$ ou $g_{i}\left(\right.$ para $\left.i=n_{0}, \ldots, n-1\right)$ multiplica o tamanho do intervalo por um fator de $K\left(\frac{\left|\delta_{i}\right|}{\left|\delta_{i-1}\right|}\right)^{\frac{1}{2}}$. As pré-imagens por ramos de $h_{n}$ ou $g_{n}$ não são tão boas e isto nos leva a considerar 2 iteradas negativas. Sendo assim faremos uma estimativa referente a $\mathcal{P}_{n}^{2 j}$ e outra para $\mathcal{P}_{n}^{2 j+1}$.

Primeiramente observamos que não ocorrem todas as possíveis 2 iteradas negativas. Os domínios de $h_{i}$ e $g_{i}\left(i=n_{0}, \ldots, n\right)$ são os intervalos $\delta_{i}$ e $\gamma_{i}$, respectivamente. Eles são encaixados do seguinte modo: $\delta_{i+1} \subset \delta_{i}$ e $\gamma_{i+1} \subset \gamma_{i}$. Disto resulta por exemplo que se $i<k$ então $\delta_{k} \cap h_{i}^{-1}\left(\delta_{k}\right)=\emptyset$ pois $\left(\delta_{i} \cup \gamma_{i}\right) \cap h_{i}\left(\delta_{k}\right)=\emptyset$. Precisaremos considerar então apenas $\left(n-n_{0}\right)\left(n-n_{0}+1\right) / 2$ pré-imagens. São pré-imagens, por exemplo, do tipo $\left(h_{k} \circ h_{i}\right)^{-1}$, com $i \leq k+1$. A pré-imagem de um intervalo $\Delta \in \mathcal{P}_{n}^{j-2}$ pelos ramos de 2 iteradas negativas deste tipo é constituída de no máximo 8 intervalos $\Delta^{\prime} \in \mathcal{P}_{n}^{j}$. Além disto se $\Delta \subset \delta_{l} \cup \gamma_{l}$ para $l \geq k$ teremos $\Delta^{\prime} \subset\left(\delta_{i} \cup \gamma_{i}\right) \backslash\left(\delta_{i+1} \cup \gamma_{i+1}\right)$. Os Lemas 5.1 e 5.2 nos permitem estimar os tamanhos. Para cada $\Delta^{\prime}$ temos que se $i \geq k$, 


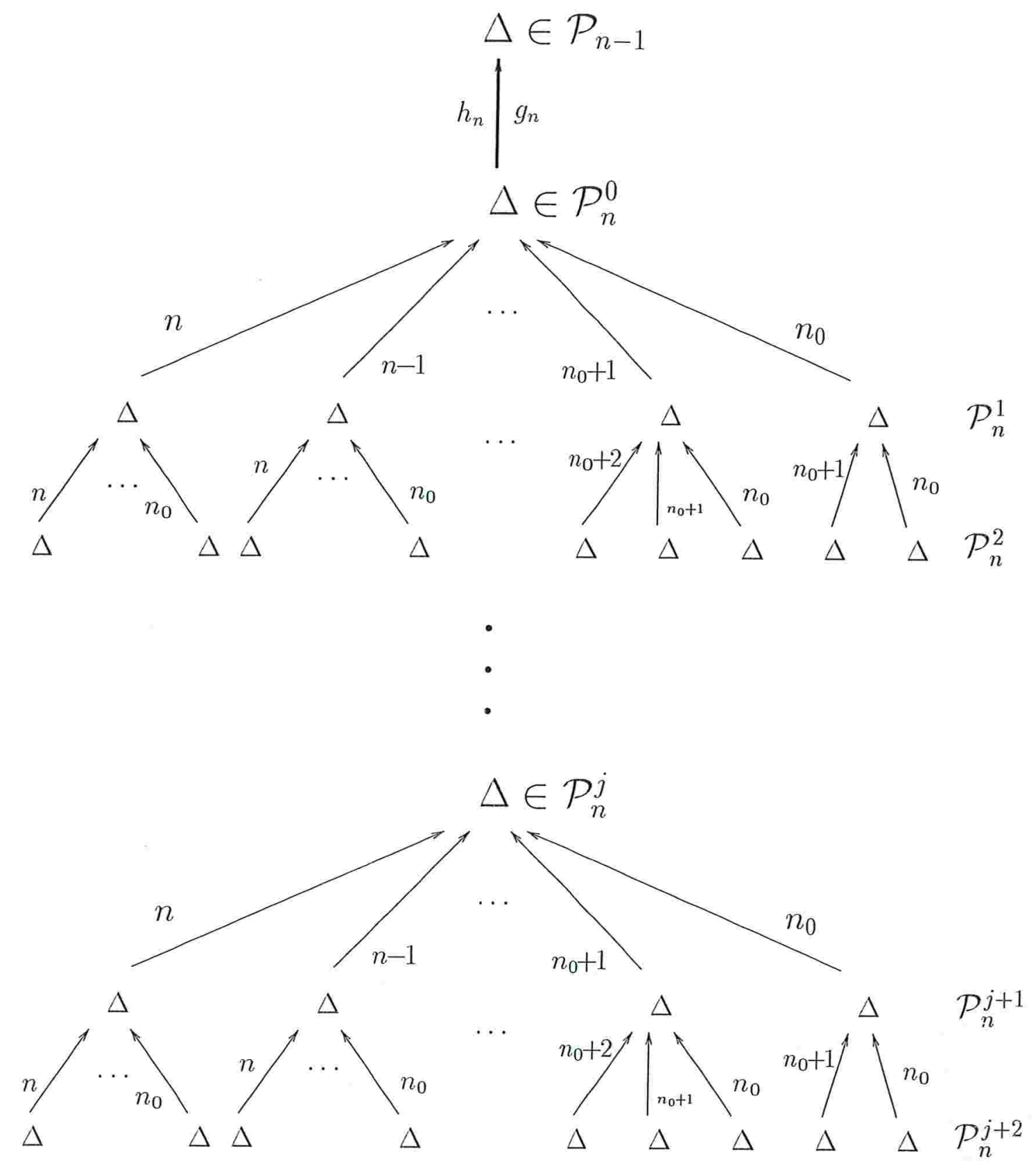

Figura 5.1: Etapas da partição $\mathcal{P}_{n}$

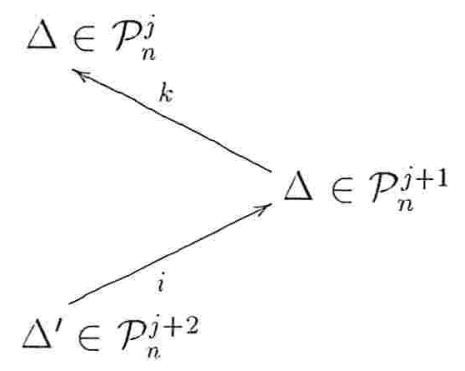




$$
\left|\Delta^{\prime}\right| \leq K^{2}\left(\frac{\left|\delta_{k}\right|}{\left|\delta_{k-1}\right|}\right)^{\frac{1}{2}}\left(\frac{\left|\delta_{i}\right|}{\left|\delta_{i-1}\right|}\right)^{\frac{1}{2}}|\Delta|
$$

No caso $i=k+1$ a estimativa é um pouco pior, a saber:

$$
\left|\Delta^{\prime}\right| \leq K^{2}\left(\frac{\left|\delta_{k}\right|}{\left|\delta_{k-1}\right|}\right)^{\frac{1}{2}}|\Delta|
$$

Também temos que $n_{\Delta^{\prime}} \leq n_{\Delta}+s_{i}+s_{k}<3 n_{\Delta}$. Concluímos que para $j \geq 2$ verifica-se que

$$
\sum_{\Delta^{\prime} \in \mathcal{P}_{n}^{j}} n_{\Delta^{\prime}}\left|\Delta^{\prime}\right| \leq 24 K^{2}\left(\sum_{i=n_{0}}^{n}\left(\frac{\left|\delta_{i}\right|}{\left|\delta_{i-1}\right|}\right)^{\frac{1}{2}} \sum_{k=i}^{n}\left(\frac{\left|\delta_{k}\right|}{\left|\delta_{k-1}\right|}\right)^{\frac{1}{2}}+\sum_{k=n_{0}}^{n-1}\left(\frac{\left|\delta_{k}\right|}{\left|\delta_{k-1}\right|}\right)^{\frac{1}{2}}\right) \sum_{\Delta \in \mathcal{P}_{n}^{j-2}} n_{\Delta}|\Delta| .
$$

Escolhemos então $n_{0}$ suficientemente grande para que

$$
b:=\sum_{k=n_{0}}^{\infty}\left(\frac{\left|\delta_{k}\right|}{\left|\delta_{k-1}\right|}\right)^{\frac{1}{2}}
$$

seja, tal que $24 K^{2}\left(b^{2}+b\right)<1 / 2$. Sendo assim temos que

$$
\sum_{\Delta^{\prime} \in \mathcal{P}_{n}} n_{\Delta^{\prime}}\left|\Delta^{\prime}\right|=\sum_{j=0}^{\infty} \sum_{\Delta^{\prime} \in \mathcal{P}_{n}^{j}} n_{\Delta^{\prime}}\left|\Delta^{\prime}\right| \leq 2\left(\sum_{\Delta \in \mathcal{P}_{n}^{0}} n_{\Delta}|\Delta|+\sum_{\Delta \in \mathcal{P}_{n}^{1}} n_{\Delta}|\Delta|\right) .
$$

O Lema 5.1 ainda permite concluir que

$$
\sum_{\Delta^{\prime} \in \mathcal{P}_{n}^{1}} n_{\Delta^{\prime}}\left|\Delta^{\prime}\right| \leq 4 K \sum_{k=n_{0}}^{n}\left(\frac{\left|\delta_{k}\right|}{\left|\delta_{k-1}\right|}\right)^{\frac{1}{2}} \sum_{\Delta \in \mathcal{P}_{n}^{0}} n_{\Delta}|\Delta| .
$$

Isto e a desigualdade 5.1 nos permitem verificar que

$$
\sum_{\Delta^{\prime} \in \mathcal{P}_{n}} n_{\Delta^{\prime}}\left|\Delta^{\prime}\right| \leq 24 K^{2}(1+8 K b)\left(\frac{\left|\delta_{n-1}\right|}{\left|\delta_{n-2}\right|}\right)^{\frac{1}{2}} \sum_{\Delta \in \mathcal{P}_{n-1}} n_{\Delta}|\Delta| .
$$

Finalmente temos que

$$
\sum_{\Delta \in \mathcal{P}} n_{\Delta}|\Delta| \leq \sum_{n=n_{0}}^{\infty}\left(24 K^{2}(1+8 K b)\left(\frac{\left|\delta_{n-1}\right|}{\left|\delta_{n-2}\right|}\right)^{\frac{1}{2}}\right)^{n-n_{0}} \sum_{\Delta \in \mathcal{P}_{n_{0}}} n_{\Delta}|\Delta| \leq L<\infty .
$$


Deste modo concluímos a finitude da média temporal.

Demonstração do Teorema C: Seja $f$ um polinômio cúbico bimodal de Fibonacci. Com o Lema 5.5 e com o Lema 5.6 garantimos a existência de uma p.i.a.c. $\nu$ para $f$, conforme anunciamos no início deste capítulo. Vamos mostrar agora que $\nu$ é érgodica e tem entropia positiva. Para isso, devemos verificar que se $X$ é um conjunto positivamente invariante e $\nu(X)>0$, então algum dos pontos críticos de $f, c$ ou $d$, é um ponto de densidade de $X$.

Observamos que $c \in \omega(x)$ ou $d \in \omega(x)$ para $x$ q.t.p. em $[0,1]$. Assim, se $X$ é positivamente invariante e $\nu(X)>0$ e, portanto $|X|>0$, dado $n \geq 1$, existe $k_{n}>0$, menor inteiro possível tal que $f^{k_{n}}(x) \in \delta_{n} \cup \gamma_{n}$. Assumimos que $f^{k_{n}}(x) \in \delta_{n}$, ou seja, $x \in \delta_{n}^{-k_{n}}$. Em $\delta_{n}^{-k_{n}}$ está definido um difeormorfismo com imagem $\delta_{n}$ e controle de distorção. Portanto,

$$
\lim _{n \rightarrow \infty} \frac{\left|X \cap \delta_{n}\right|}{\left|\delta_{n}\right|}=1
$$

e $c$ é um ponto de densidade para $X$ em relação à medida de Lebesgue. Em particular, $c$ ou $d$ é um ponto de densidade para o suporte da medida $\nu$. Se $\nu$ tivesse entropia nula, $f$ seria invertível em $\nu$-q.t.p. (veja [Le]). Então o suporte de $\nu$ deveria ter densidade no máximo $1 / 2$ em cada ponto crítico, o que contradiz o fato de $c$ ou $d$ ser ponto de densidade do suporte de $\nu$. Logo, $\nu$ tem entropia positiva.

Para mostrar que $\nu$ é ergódica, observamos que dados dois conjuntos positivamente invariantes $X$ e $Y \operatorname{com} \nu(X \cap Y)=0$, então um dos dois tem medida nula. De fato, se os dois tivessem medida positiva, $c$ ou $d$ seria ponto de densidade de ambos. Então $X$ ou $Y$ deve ter medida nula e $\nu$ é ergódica. 


\section{Referências Bibliográficas}

[BKNSt] Bruin, H., Keller, G., Nowicki, T., van Striem, S.: Wild Cantor attractors exist - Ann. of Math.(2), 143, (1996) $n^{\circ}$ 1, 97-130.

[BSt] Bruin, H. \& van Strien, S. : Existence of acips for multimodal maps - Global Analysis of Dynamical Systens, 433-447, Inst. Phys., Bristol (2001).

[GS] Graczyk, J. \& Świątek, G.: Induced expansion for quadratic polynomials -, Ann. Scient. Éc. Norm. Sup. 29 (1996), 399-482.

[G] Guckenheimer,J.:Limit sets of S-unimodal maps with entropy zero - Commun. Math. Phys., 110, (1987), 655-659.

[HK1] Hofbauer, F \& Keller, G.: Quadratic maps without asymptotic measures -, Commun. Math. Phys. 127, (1990) n² 319-337.

[HK2] Hofbauer, F. \& Keller, G.: Some remarks about recent results on $S$ unimodal maps - Ann. de l'Institut Henri Poincaré, Physique Théorique, 53 (1990), 413-425

[JS] Jakobson, M. \& Swiatek, G.: Metric Properties of non-renormalizable Sunimodal maps. Part I - Induced expansion and invariant measures. Ergod. Th \& Dynam. Sys. 14 (1994).

[J] Jonhson, S. D.: Singular measures without restrictive intervals -. Commun. Math. Phys. 110 185-190 (1987).

[Le] Ledrappier, F. : Some properties of absolutely continuos invariant measures for maps with expoent zero -Ergod. Th. \& Dynam. Sys. 1, 77-93 (1981).

[L] Lyubich, M. Ergodic theory for smoth one-dimensional dynamical systens SUNY - preprint 1991/11.

[LM] Lyubich, M. Yu. \& Milnor, J.: The Fibonacci Unimodal Maps -J. Americ. Math. Soc.6, (1993), no 2, 425-457. 
[Ma] Mañé, R.: Hyperbolicity, sinks, and measure en one-dimensional dynamics - Commun. Math. Phys., 100, (1985), 495-524 e Erratum - Commun. Math. Phys., 112, (1987), 721-724.

[MN] Martens, M. \& Nowicki, T.: Invariant Measures for Typical Quadratic Maps - Asterisque 261 239-252 (2000).

[MSt] de Melo, W. \& van Strien, S.: One-Dimensional Dynamics. Springer, BerlinHeidelberg - New York, (1991).

[M] Milnor, J.: On the concept of attractor -Commun. Math. Phys., 99, (1985) 177-195 e On the Concept of attractor: correction and remarks - Commun. Math. Phys., 102, (1985) 517-519.

[NSt] Nowicki,T. \& van Strien, S.: Absolutely continuos invariant measures under a summability condition - Invent. Math. 105, 123-136 (1991).

[Si] Singer, D.:Stable orbits and bifurcations of maps of the interval - SIAM J. Appl. Math. 35, 260-267

[StV] van Strien, S \& Vargas, E.: Real Bounds, ergodicity and negative Schwarzian for multimodal maps - submitted for publication (2002).

[SV] Świątek, G. \& Vargas, E.: Decay of geometry in the cubic family - Ergod. Th. \& Dynam. Sys. 18, (1998), 1311-1329. 